\title{
Transitive Mate Preferences
}

\author{
Hongyi Wang and Zhilin He \\ East China Normal University \\ Lisheng $\mathrm{He}$ \\ Shanghai University
}

\begin{abstract}
Author Note
H.W. was supported by National Natural Science Foundation of China (71901095), China Postdoctoral Science Foundation (2018M632052) and Shanghai Pujiang Program (18PJC034). L.H. was supported by Shanghai Pujiang Program (2020PJC102) and the Fundamental Research Funds for the Central Universities (2020114083). Portions of the research use the oriental face database collected under the research of the Artificial Intelligence and Robotics (AI\&R).
\end{abstract}

Correspondence concerning this article should be addressed to Lisheng He at SILC Business School, Shanghai University, Shanghai, China (felix8.he@gmail.com). 


\begin{abstract}
Rational mate choices are central to individual happiness and collective social goods. Yet, few studies assess mate choice rationality from the decision-theoretic perspective. Here we present an experimental test of rationality in human mate preferences through the lens of transitivity, a fundamental hallmark of rational decision-making. In the experiment, participants made repeated binary choices between pairs of potential romantic partners in both short-term and long-term mating contexts. We tested the transitivity of mate preferences by systematically comparing four prominent transitive models with four models that allow for intransitive preferences on the choice data. Overall, all transitive models provided better accounts than the intransitive models in Bayesian model selection and strong stochastic transitivity (SST), the most restrictive transitive model, outperformed other transitive models. On the individual level, participants rarely displayed intransitive cycles and most of them were best described by transitive models in Bayesian model selection. Our paper presents a systematic evaluation of transitivity in mate preferences and sheds new light on our understanding of human mating behavior.
\end{abstract}

Keywords: mate preference, rational choice, transitivity, Bayesian model selection 


\section{Introduction}

Mate choice is one of the most important choices for our lives. It plays significant roles in individual happiness, as well as social welfare (Diener \& Seligman, 2002). How do we choose romantic partners? What qualities and quantities do we seek in potential partners? Researchers have long been interested in the characteristics that we favor in romantic partners, as the answers to these questions are crucial to our understanding of human mating behavior (Hill, 1945; Buss, 1989).

Mate choice is influenced by a wide range of factors, such as personal traits (e.g. attractiveness, personality and similarity), mutual affect (e.g., love), social status and financial prospects (Buss et al., 2001). Of all the attributes that we consider in potential mates, physical attractiveness and financial resources are among the most influential ones. Both men and women assign high priorities to them when considering romantic partners (Li et al., 2002; Li \& Kenrick, 2006; Todd et al., 2007; Zhang et al., 2019). The importance of these two attributes is almost universal and has been increasing steadily in the last several decades (Buss et al., 2001; Walter et al., 2020).

\section{Evolutionary and social determinants of mate preferences}

Despite the global consensus, people may weight physical attractiveness and financial resources differently in different in different contexts. Specifically, men typically more heavily weight physical attractiveness than women do, and women tend to more heavily weight financial resources than men do (Buss, 1989; Buss \& Barnes, 1986; Buss et al., 2001; Feingold, 1990; 1992). The sex differences are 
typically attributed to socioeconomic and evolutionary reasons. Physical attractiveness is widely accepted to be a premium in social relationships and, additionally, women's physical attractiveness, from an evolutionary perspective, is thought to signal their reproductive capacity (Buss \& Schmitt, 1993; Gangestad \& Simpson, 2000; Maestripieri et al., 2017). Conversely, financial resources (e.g., resource-related attributes such as ambition, earning capacity, social status) are valued heavily by women likely because women contribute more to the obligatory parental investment (i.e., pregnancy and lactation) than men do (Trivers, 1972) and resources provided by men can be beneficial to both the women and their offspring (Buss \& Schmitt, 1993; Kenrick \& Keefe, 1992). Thus, men and women appear to act in a way to rationally maximize their own benefits in mating behavior.

Their weights may also differ in different mating contexts. Women tend to value financial resources less in short-term partners than in long-term partners because financial resources are thought to be less important in short-term relationships. Rather, they place greater emphasis on physical attractiveness for shortterm partner selection, since it might be more beneficial to focus on good genes (as displayed by cues such as physical condition) as this could be heritable to their offspring (Buss \& Schmitt, 1993; Gangestad \& Simpson, 2000; Kenrick et al., 1993; Li \& Kenrick, 2006; Li et al., 2011). Besides, in societies with greater gender equality, the sex differences of mate preferences are likely to be smaller (Eagly \& Wood, 1999; Walter et al., 2020). It has been argued that the adaption to mating 
contexts and social environments signals the ecological and social rationality of mate preferences (Lenton et al., 2013; Todd et al., 2012).

\section{Transitivity: Decision-theoretic rationality}

The aforementioned social and evolutionary accounts provide compelling interpretations of the findings that men and women have different mate preferences and that they adjust their preferences adaptively upon different mating contexts and social environments. The rationality of mate choice, however, can be also assessed within a decision-theoretic framework. Axiomatic decision theories examine choice rationality via the structural consistency between choices, setting aside the weighting of attributes that determines mate values, and assert that rational choice should follow these principles. In behavioral decision research, decision-theoretic testing has proven highly valuable and has provoked fruitful research programs on the psychology of decision-making (Tversky, 1969; Kahneman \& Tversky, 1979; Huber et al., 1982; Simonson, 1989; Regenwetter et al., 2011).

Transitivity is one of the most important decision-theoretic principles of rationality (Fishburn, 1973; Luce, 1959). It means that, for any given triplet $\{A, B, C\}$, if one prefers $A$ to $B$ and $B$ to $C$, she should prefer $A$ to $C$. This transitive preference can be written as $A>B>C$. Otherwise, if transitivity is violated, people may exhibit irrational and cyclic choice patterns, such as $A>B, B>C$ but $C>A$. Someone who maximizes their profits in the choices should not display the cyclic patterns and thus should follow the transitivity axiom. In light of the theories that suggest that both men's and women's mate preferences are shaped in a way to 
maximize their own interests, transitivity testing is a uniquely powerful tool for evaluating the rationality of mate preferences.

Transitivity is closely related to the distinction between the compensatory and non-compensatory strategies in mate choice. Compensatory strategies refer to the decision processes that weigh all the relevant attributes and assign the weight to each attribute, allowing a low value on one attribute to compensated by a high value on another attribute. Compensatory strategies are typically consistent with transitive preferences. For example, the weighted-additive model assumes that individuals assess all relevant cues, multiply by the weight that the individuals assign to the cue and choose the one with the highest summed value (Brandner et al., 2020; Buss \& Schmitt, 1993; Miller \& Todd, 1998). The Euclidean Distance model proposed by Conroy-Beam (Conroy-Beam, 2018; Conroy-Beam \& Buss, 2016; 2017) computes the distance from actual trait values to ideal trait values in a multidimensional space and the option with smaller distance has a higher mate value.

In contrast, non-compensatory strategies may violate the principle of transitivity. For example, the lexicographic rules assume that decision-makers consider only one attribute at a time (Tversky, 1969). A decision is reached as soon as the attribute under consideration favors one option against others, disregarding information on other attributes. Several heuristic models, such as Take-the-Best (Gigerenzer \& Goldstein, 1996; Miller \& Todd, 1998) and Avoid-the-Worst (Grammer et al., 2002) are instances of lexicographic rules. Another noncompensatory example is the sequential aspiration-level model (Miller \& Todd, 
1988). This model assumes an individual assesses a potential mate's trait values sequentially and pursues further courtship only if the potential mate's trait value exceeds the minimum ideal value. These non-compensatory strategies may lead to intransitive preferences.

Transitivity is also essential to the popular idea of mate value, a procedure that combines different attributes in mate choice into an overall numeric value (Symons, 1979; see Simao \& Todd, 2002; Todd \& Miller, 1999 for applications). Transitivity is the necessary condition for integrating different attributes into an overall unidimensional value because the unidimensional values are transitive themselves. If the transitivity of mate preference does not hold, it may be problematic to assign a unidimensional value to each potential mating partner in practice.

\section{Experimental test of transitivity}

Despite its theoretical and practical significance, few studies have examined the transitivity of human mate preferences. Very recently, Brandner et al. (2020) compared a set of compensatory $v s$. non-compensatory strategies in mate choice and found that compensatory strategies captured human mate choice data much better than non-compensatory strategies. Although they did not directly test transitivity, their results suggested that human mate preferences might be transitive. Another study by Hatz et al. (2020) directly tested the transitivity of risky sexual decision-making. They formulated risky sexual decisions using facial attractiveness and the probability of contracting a sexually transmitted infection. Their model comparison focused on the Weak Order Mixture Model of Regenwetter and Davis-Stober (2012) as a stochastic 
model of transitivity and the lexicographic semiorder mixture model of Davis-Stober (2012) as the intransitive competitor model and suggested that the transitive model fits the data better than the intransitive one.

Previous work suggests that mate preferences are probably transitive. However, the conclusion regarding transitivity may be sensitive to the formulation of the transitive models. For example, Tversky's (1969) pioneering work on the transitivity of risk preferences suggested that people may show intransitive preferences in gamble choices. Similarly, Roelofsma and Read (2000) reported intransitivity in intertemporal choice, a decision scenario where people choose between payoffs available at different time points. Recent analyses, however, suggested that the conclusions regarding transitivity may depend on the stochasticity assumption of the transitive model. Some seemingly intransitive patterns may be due to the stochasticity of preferential choice that was not considered in the analyses (see Birnbaum \& Gutierrez, 2007; Birnbaum \& Schmidt, 2008; 2010; Cavagnaro \& DavisStober, 2014; Regenwetter et al., 2010; 2011).

The conclusion regarding transitivity is also sensitive to the competitor models that allow for intransitive preferences. For example, in a test of transitivity of intertemporal choice, Dai (2017) suggested that although transitive models may explain data better than the saturated model that allowed for any combination of choice probabilities, the data could be even better explained by well-tuned intransitive models other than the overly flexible saturated model. Thus, it is important to involve 
different forms of transitive and intransitive models for a rigorous test of transitivity, revealing the cognitive principles underlying mate choice.

\section{Current study}

This paper presents a direct test of transitivity in human mate preferences.

Like Brandner et al. (2020), we were interested in how people integrate different cues in partner selection in the typical mate choice settings. According to the extensive evolutionary and social psychology literature, physical attractiveness and financial resources are arguably the most pervasive determinants of mate values. We thus created profiles of potential partners varying in physical attractiveness and financial resources for heterosexual women and men. We also considered two within-subject choice conditions, short-term and long-term partner selection, to test preference transitivity in different mating contexts.

Like Hatz et al. (2020), we directly compared transitive models with models that allowed for intransitive preferences. To address the sensitivity to the specification of transitive and intransitive models, we systematically tested four prominent transitive models and compared them with four models that allowed for intransitive preferences. Three transitive models (i.e., weak stochastic transitivity, medium stochastic transitivity, and strong stochastic transitivity) assume that participants have a fixed order of preferences but allow for unsystematic errors of selecting the less preferred option. The predicted choice probability is determined by the preference order and the allowed probability of committing an error. The fourth transitive model, mixture model of transitive preference (MMTP), allows for the fluctuation of 
preference orders over time, according to which the predicted choice probability is the average of all possible preference orders.

In line with previous studies (e.g., Davis-Stober, 2012; Hatz et al., 2020), we considered lexicographic semiorder models as the main intransitive competitor models that allowed both transitive and intransitive preferences. To explore different sources of randomness, we specified three variants of the lexicographic semiorder model with different stochastic assumptions. The lexicographic semiorder models resemble some popular heuristic models of mate choice, such as Take-the-Best and Avoid-the-Worst (Gigerenzer \& Goldstein, 1996; Grammer et al., 2002; Miller \& Todd, 1998). Finally, we also involved a fourth intransitive model that allowed for full flexibility of choice probabilities (i.e. the saturated model) in the model comparison as the benchmark model.

\section{Methods}

\section{Participants}

Fifty-six heterosexual women $\left(M_{\mathrm{age}}=20.00, S D_{\mathrm{age}}=2.09\right)$ and 51 heterosexual men $\left(M_{\text {age }}=20.65, S D_{\text {age }}=2.51\right)$ participated in the study. All the participants were students at East China Normal University, Shanghai, China. The study was approved by the Committee of Human Research Protection at East China Normal University.

\section{Stimuli}

We designed two sets of profiles, one Main set and one Alternative set. Each set consists of $m=5$ profiles of potential mates. Each profile contained information on two attributes: physical attractiveness (indicated by a face image) and financial 
resources (indicated by an annual income). Our construction of stimuli was primarily inspired by Tversky's (1969) classic design for testing transitivity in risky choice. Regenwetter et al. (2010, 2011) also suggest that one of the transitive models (i.e., the mixture model of transitive preference as introduced below) cannot be properly defined and fit to experimental data easily unless $m \leq 5$.

Following previous studies (e.g., Brandner et al., 2020; Hatz et al., 2020), we used face images to represent physical attractiveness. Face images were selected from the Oriental Face Database (http://gr.xjtu.edu.cn/web/jianyi/english-version). We recruited an independent group of participants (21 heterosexual men and 17 heterosexual women) to rate the physical attractiveness of opposite-sex face images. Ten faces were selected to represent different levels of physical attractiveness based on the average rating scores (from the independent group of participants). Since people might judge physical attractiveness differently (Hehman et al., 2017), we asked participants to rank the face images on physical attractiveness and then paired the image faces with the annual income adaptively for each participant (denoted as $A$, $B, C, D$, and $E$, with $A$ as the most attractive and $E$ the least attractive). The annual incomes also had five levels and were set at $¥ 90,000$, $¥ 120,000, ¥ 150,000$, $¥ 180,000$, and $¥ 210,000$ per year. At the moment of the experiment, ¥1,000 (Chinese Yuan) was approximately worth $\$ 142$ (US Dollar). We chose annual incomes in the middle range, without extremely high/low values.

In the Main set, physical attractiveness and financial resources were set negatively correlated (i.e., the more attractive one had a lower annual income). 
Typically, both women and men have a preference for attractive partners and partners with good financial resources (Buss, 1989; Buss et al, 2001; Feingold, 1990; 1992) and thus they needed to make trade-offs between these two attributes in the binary choices. In the Alternative set, the two attributes were set positively correlated (i.e., the more attractive one also had a higher annual income). We included this design set in order to catch the trade-offs if participants perceived financial resources as a negative attribute.

\section{Procedure}

Participants first rank-ordered the two sets of five face images respectively. With each set, they sorted the images from the most attractive to the least attractive by dragging the images onscreen. They were able to sort the images as many times as they wanted before submitting their final rank orders. This rank order was in turn used to create profiles. For the Main set, the most attractive face image was paired with the lowest salary $¥ 90,000$; the second most attractive image was paired with the second lowest salary $¥ 120,000$ and so on ...; the least attractive face image was paired with the highest salary $¥ 210,000$. For the Alternative set, the most attractive face image was paired with $¥ 210,000$ and the least attractive face image was paired with $¥ 90,000$, and so on.

In the mate choice task, participants made binary choices in pairs of potential romantic partners' profiles. Ten unique binary choices were created from the five profiles for each set, making up a total of 20 unique binary choices. The 20 binary choices were repeated 10 times, with each repetition as a separate block. Participants 
were allowed to take a self-paced break between blocks. Note that the choices within each block were fully randomized. The two design sets were mixed within each block to reduce the memorability of past choices, allowing us to obtain repeated but independent choice frequencies across blocks (see Tversky, 1969 for a similar manipulation).

To test different mating contexts, each participant performed the mate choice tasks twice; once for choosing long-term (i.e., marriage) partners and once for choosing short-term (i.e., casual sex) partners. Participants completed all the choices in one mating context before moving on to the other. The order in which participants did these two tasks was randomized across participants.

\section{Models}

\section{Transitive models}

For a rigorous test of transitivity, we considered four stochastic models of transitivity: weak stochastic transitivity (WST), medium stochastic transitivity (MST), strong stochastic transitivity (SST, see Falahatgar et al. 2018 for a recent overview), and mixture model of transitive preference (MMTP) (Regenwetter et al. 2011). The first three models assumed that individuals would hold fixed preference orders but may occasionally make errors of choosing the less preferred option, while MMTP allowed for fluctuation of preferences from moment to moment such that different decisions at different time points reflect different preference orders. The four transitive models can be written precisely as follows: 
Weak stochastic transitivity. $\forall$ (distinct) $x, y, z \in \mathcal{S}:$ if $\operatorname{Pr}[x ; y] \geq$ $\frac{1}{2}$ and $\operatorname{Pr}[y ; z] \geq \frac{1}{2}$, then $\operatorname{Pr}[x ; z] \geq \frac{1}{2}$, where $\mathcal{S}=\{A, B, C, D, E\}$ is the full set of options. Note that $x, y$ or $z$ here represents any element from $\mathcal{S}=\{A, B, C, D, E\}$. They are treated in the same way as for $A, B, C, D$ or $E$.

Medium stochastic transitivity. $\forall$ (distinct) $x, y, z \in \mathcal{S}$ : if $\operatorname{Pr}[x ; y] \geq$ $\frac{1}{2}$ and $\operatorname{Pr}[y ; z] \geq \frac{1}{2}$, then $\operatorname{Pr}[x ; z] \geq \min \{\operatorname{Pr}[x ; y], \operatorname{Pr}[y ; z]\}$, where $\mathcal{S}=$ $\{A, B, C, D, E\}$ is the full set of options.

Strong stochastic transitivity. $\forall$ (distinct) $x, y, z \in \mathcal{S}:$ if $\operatorname{Pr}[x ; y] \geq$ $\frac{1}{2}$ and $\operatorname{Pr}[y ; z] \geq \frac{1}{2}$, then $\operatorname{Pr}[x ; z] \geq \max \{\operatorname{Pr}[x ; y], \operatorname{Pr}[y ; z]\}$, where $\mathcal{S}=$ $\{A, B, C, D, E\}$ is the full set of options.

Mixture model of transitive preference. $\forall$ (distinct) $x, y, z \in \mathcal{S}: \operatorname{Pr}[x ; y]+$ $\operatorname{Pr}[y ; z]-\operatorname{Pr}[x ; z] \leq 1$, where $\mathcal{S}=\{A, B, C, D, E\}$ is the full set of options (see Regenwetter et al. 2011 for a detailed discussion of the mixture model of transitive preferences).

The four transitive models differed in model flexibility. This can be illustrated by the choice probability space each model allows. We ran a Monte Carlo analysis to demonstrate this. The five options formed a 10-dimensional choice-probability space, with each dimension representing the choice probability between one pair of options (ranging from 0 to 1). We drew 10 billion uniform samples from the full space (i.e., the saturated model), tested whether each of the samples was consistent with the models' predictions, and then estimated the proportion of the saturated model's predictions that were consistent with each of the models above. 
As in the "Standard" column of Table 1, WST was the most flexible transitive model, allowing for $11.7 \%$ of the saturated model. SST, by contrast, was the most parsimonious one, allows for less than $0.01 \%$ of the saturated model. MST and MMTP lay in between. Overall, the small proportions suggested that the test of these models against the saturated model was highly viable.

\section{Table 1}

The allowed proportions of the saturated model space by the substantive models. The "Standard" column corresponds to the standard version of the transitive and LS models and the "Constrained" column corresponds to the models with the additional preference order constraint of $A>B>C>D>E$.

\begin{tabular}{ccc}
\hline \multirow{2}{*}{ Model } & Proportion of the saturated model allowed by each model \\
\cline { 2 - 3 } & Standard & Constrained \\
\hline WST & $1.17 \times 10^{-1}$ & $9.75 \times 10^{-4}$ \\
MST & $5.12 \times 10^{-3}$ & $4.26 \times 10^{-5}$ \\
SST & $2.42 \times 10^{-5}$ & $2.05 \times 10^{-7}$ \\
MMTP & $4.80 \times 10^{-2}$ & $2.39 \times 10^{-4}$ \\
LSEM01 & $7.82 \times 10^{-3}$ & $9.77 \times 10^{-4}$ \\
LSEM25 & $1.73 \times 10^{-2}$ & $3.97 \times 10^{-3}$ \\
LSMM & $4.24 \times 10^{-4}$ & $4.17 \times 10^{-7}$ \\
\hline
\end{tabular}


We considered four models that allowed for intransitive preferences. The first three models were variants of the lexicographic semiorder model (LS). The lexicographic semiorder model was an influential cognitive model that allowed for intransitive preferences (Tversky, 1969). LS held an attribute-wise evaluation rule and implemented a threshold mechanism that permitted intransitive preferences. The fourth intransitive model was the saturated model that allowed for any choice probabilities. It was the most flexible model in the context of two-alternative forced binary choice.

Lexicographic semiorder error models. LS models can be equipped with a choice-error specification. To illustrate, consider a choice between $A$ and $B$ (where $A$ is more attractive, but $B$ has a higher income). One may first compare the attractiveness between $A$ and $B$. If she perceives $A$ as being more attractive than $B$ and the difference in attractiveness reaches a threshold, she is likely to choose $A$ over $B$. In a probabilistic term, that means $\frac{1}{2} \leq \operatorname{Pr}[A ; B] \leq 1$. If the difference does not reach the threshold, she continues to evaluate the annual income. If the difference along income reaches a threshold, then she is likely to choose $B$ over $A$, with $0 \leq$ $\operatorname{Pr}[A ; B] \leq \frac{1}{2}$. If the difference along the annual income does not reach the threshold either, she is indifferent between $A$ and $B$. LS models also allow participants to compare annual incomes first. If someone perceives that the advantage of $B$ 's annual income over $A$ 's annual income reaches a threshold, she is likely to choose $B$ with $0 \leq \operatorname{Pr}[A ; B] \leq \frac{1}{2}$. If not, she continues to evaluate physical attractiveness. If $A$ 's attractiveness surpasses $B$ 's and this difference reaches a threshold, she is likely to 
choose $A$ over $B$, with $\frac{1}{2} \leq \operatorname{Pr}[A ; B] \leq 1$. Otherwise, she is indifferent between $A$ and $B$.

Different threshold values for physical attractiveness and annual income created potentially different preference semiorders for the LS models. As attractiveness was represented by rank orders, the threshold for attractiveness, defined as the difference between rank orders, is on an ordinal scale. The threshold can take five different levels: 1 (meaning that all of the pairwise differences reach the threshold), 2, 3, 4, 5 (meaning that none of the pairwise differences reaches the threshold). Likewise, the threshold for annual income takes five different levels (i.e. $¥ 1 \sim 29,999, ¥ 30,000 \sim 59,999, ¥ 60,000 \sim 89,999, ¥ 90,000 \sim 119,999, ¥ 120,000$ or more). That is because, any threshold value between $¥ 1$ and $¥ 29,999$, for example, makes the same prediction regarding the whole set of options, $\{A, B, C, D, E\}$. Any threshold value between $¥ 30,000$ and $¥ 59,999$ also makes the same prediction, and so on.

The combination of the threshold values along the two attributes and the two sequential orders of evaluation (evaluating attractiveness first or annual income first) created 21 non-redundant preference orders or semiorders for the LS models (see Supplemental Table S1 for the list of non-redundant orders or semiorders). For any distinct $x, y \in\{A, B, C, D, E\}$, if $x$ is preferred to $y, \frac{1}{2} \leq \operatorname{Pr}[x ; y] \leq 1$. If $y$ is preferred to $x, 0 \leq \operatorname{Pr}[x ; y] \leq \frac{1}{2}$. The ideal choice probability specification for the "indifference between $x$ and $y$ " is $\operatorname{Pr}[A ; B]=\frac{1}{2}$. However, the volume-based Bayesian model selection method, as detailed later, required us to transform the 
equality into "about equality" $\operatorname{Pr}[A ; B] \approx \frac{1}{2}$ (see Klugkist \& Hoijtink, 2007). Here, we explored two versions of the "about equality" specifications. In one version, we allowed for a very small deviation of choice probability and specified $0.49 \leq$ $\operatorname{Pr}[A ; B] \leq 0.51$, referred to as $L S E M 01$, such that the allowed probability values were close to $\frac{1}{2}$ (“ 01 " refers to the allowed deviation of 0.01 from $\frac{1}{2}$ ). In the other version, we specified $\frac{1}{4} \leq \operatorname{Pr}[A ; B] \leq \frac{3}{4}$, to make the width of this "about equality" equal to the error width of other preference orders between $A$ and $B$ (e.g., $0 \leq$ $\operatorname{Pr}[x ; y] \leq \frac{1}{2}$, see Guo, 2018 for a similar specification). We referred to the latter as LSEM25 (" 25 " refers to the allowed deviation of 0.25 from $\frac{1}{2}$ ). For either LSEM01 or LSEM25, the predicted region was the union of the 21 preference orders' or semiorders' predicted regions. As in the "Standard" column of Table 1, LSEM01 allows for $0.78 \%$ of saturated model while LSEM 25 is more flexible, allowing for $1.73 \%$ of the saturated model.

Lexicographic semiorder mixture model. LS models can also be equipped with mixture-preference specifications (referred to as lexicographic semiorder mixture model or LSMM). In the LSMM model, decision-makers are assumed to hold a preference semiorder at each point of time, but the preference semiorder may vary from moment to moment. Consequently, the overall choice probabilities are governed by the average of all states of preference orders or semiorders. We follow DavisStober (2012) to formally characterize LSMM's predicted region (see Davis-Stober et al., 2015; 2019; Hatz et al., 2020 for similar applications). As for lexicographic semiorder error models, we considered two possible procedures of decision making in 
LSMM, allowing decision-makers to treat either attractiveness and annual income as the primary attribute respectively.

Saturated model. Finally, we involved the saturated model that allowed for all possible pairwise choice probabilities (see also Sopher \& Gigliotti, 1993; Regenwetter et al., 2011). The saturated model served as a benchmark model. If someone's data were best described by the saturated model, that means none of the transitive and lexicographic semiorder models provided an adequate account for the data. It is important to note that the intransitive models, including the lexicographic semiorder models and the saturated model, do not exclude transitive preferences. Rather, they allow for both transitive and intransitive choice patterns.

\section{Constrained models}

As noted earlier, although most participants would prefer attractive to unattractive partners and prefer high-income to low-income partners, we expected that some might not prefer high-income to low-income partners, especially in short-term mating contexts. To account for these individual differences, we designed the Main and Alternative sets to increase the diagnosticity of our transitivity test. To unravel the diagnosticity of each stimulus set at the individual level, we defined another set of transitive and LS models, by introducing the preference order constraint of $A>B>$ $C>D>E$. Mathematically, the constraint can be expressed as:

$$
\begin{aligned}
& \operatorname{Pr}[A ; B] \geq \frac{1}{2} \& \\
& \operatorname{Pr}[A ; C] \geq \frac{1}{2} \& \\
& \operatorname{Pr}[A ; D] \geq \frac{1}{2} \&
\end{aligned}
$$




$$
\begin{aligned}
& \operatorname{Pr}[A ; E] \geq \frac{1}{2} \& \\
& \operatorname{Pr}[B ; C] \geq \frac{1}{2} \& \\
& \operatorname{Pr}[B ; D] \geq \frac{1}{2} \& \\
& \operatorname{Pr}[B ; E] \geq \frac{1}{2} \& \\
& \operatorname{Pr}[C ; D] \geq \frac{1}{2} \& \\
& \operatorname{Pr}[C ; E] \geq \frac{1}{2} \& \\
& \operatorname{Pr}[D ; E] \geq \frac{1}{2} .
\end{aligned}
$$

We applied the constraint to both the transitive and LS models. The constrained models are more restrictive than the standard models as in the "Constrained" column of Table 1. For example, the constrained WST/MST/SST models only allow for $1 / 120$ of the standard WST/MST/SST models' predicted regions.

The combination of stimulus and model designs allowed us to identify the diagnostic design set for each participant. For the majority of participants who preferred high income to low income in partner selection, the options in the Main set offered a valid tradeoff between attractiveness and financial income, facilitating a strong test of transitivity. They were likely to follow the constrained models with the Alternative set because in the Alternative set, $A$ was both more attractive and more affluent than $B, B$ was both more attractive and affluent than $C$, etc. On the other hand, for those who preferred low income to high income in partner selection, the Alternative set offered a valid tradeoff between options, facilitating a strong test of transitivity. Likewise, they were likely to follow the constrained models in the Main 
set. We ultimately used the constrained models to precisely identify the data that was diagnostic for transitivity testing, while heterogeneous attribute weighting was allowed.

\section{Bayesian model selection}

We used the Bayesian approach to penalize models for their flexibility in model comparison (see Supplemental S2 for a model recovery analysis). Each of the models had ten free parameters, corresponding to the probabilities of choosing the more attractive option, $p_{i}$, in each of the 10 unique choice pairs. The log-likelihood for one dataset (consisting of 10 unique choice pairs) is $\log L=\log \left(\left(\begin{array}{c}N_{i} \\ f_{i}\end{array}\right)\right)+$ $f_{i} \log \left(p_{i}\right)+\left(N_{i}-f_{i}\right) \log \left(1-p_{i}\right)$, where $f_{i}$ is the frequency of choosing the more attractive option in pair $i, N_{i}$ is the number of choice repetitions of pair $i$ (which was always 10 in the experiment) and $\left(\begin{array}{c}N_{i} \\ f_{i}\end{array}\right)$ is the binomial coefficient. For each model, we assumed a uniform prior distribution of $p_{i}$ over the allowed region for the Bayesian analysis. Note that we fit each design for each participant in each condition independently.

Directly fitting these models to the choice data for a Bayesian model selection was rather complicated. Fortunately, all the models in Table 1 were nested in the saturated model. Because of the nested nature, we carried out Bayesian model selection by drawing posterior samples from the saturated model only and evaluating the match/mismatch between the posterior samples and each nested model (Klugkist \& Hoijtink, 2007; see similar applications in Cavagnaro \& Davis-Stober, 2014; Dai, 
2017). Specifically, we estimated a Bayes factor between a nested model $M$ and the saturated model $S$ :

$B F_{M, S}=\frac{c_{M}(p(\boldsymbol{\theta} \mid S, D))}{c_{M}(p(\boldsymbol{\theta} \mid S))}$

where $c_{M}(p(\boldsymbol{\theta} \mid S))$ is the proportion of the saturated model's prior in agreement with the constrained model $M$ (as in Table 1$)$, and $c_{M}(p(\boldsymbol{\theta} \mid S, D))$ is the proportion of the saturated model's posterior in agreement with $M$. Intuitively, the Bayes factor can be interpreted as the extent to which the data has swayed the prior away from or towards the regions in agreement with $M$.

We drew posterior samples from the saturated models using Stan (Stan Development Team, 2020). For each fit to a dataset (i.e., 10 binary choices $\times 10$ repetitions), five independent chains were simulated. Each chain consists of two million formal samples following a 1,000-sample burn-in period. We then evaluated the posterior samples to determine the proportion that agreed with the nested model $M, c_{M}(p(\boldsymbol{\theta} \mid S, D))$. The Bayes factor between two models can be calculated as $B F_{M_{1}, M_{2}}=\frac{B F_{M_{1}, S}}{B F_{M_{2}, S}}$

The above computational analyses were run twice independently for convergence check. The two independent runs identified the same best-performing model for $90.4 \%$ of the datasets. In cases where the two runs identified different best models, the Bayes factors between the two were always close to 1 in both repetitions, with values between 0.87 and 1.08 , suggesting that these "inconsistencies" were indeed because the two models explained the data almost equally well. The deviation of posterior model probabilities between the two runs were small, ranging from 
0.00002 to $0.018($ mean $=0.0016$, median $=0.0009)$ across participants and conditions. Overall, these statistics indicated good convergence across repetitions. The model comparison results reported below were based on the average of the two independent runs. Note that in the following, we used the criterion of BF $>1$ to identify the best-performing model in Bayesian model selection unless specified otherwise.

\section{Results}

\section{Aggregate choice proportions}

We firstly examined the aggregate choice data in different conditions. Table 2 summarizes the overall choice proportions (see Supplemental Table S3 for an itemwise decomposition). With the Main set, men chose the attractive-but-low-income profiles more frequently than women $\operatorname{did}\left(F(1,105)=16.03, p<.001, \eta_{G}^{2}=.11\right)$, which was consistent with the idea that men assigned more weight to physical attractiveness but less weight to financial resources than women did. Participants chose attractivebut-low-income profiles more frequently for short-term partners than for long-term partners $\left(F(1,105)=71.69, p<.001, \eta_{G}^{2}=.13\right)$ and the difference between short- and long-term partners was more pronounced for women than for men $(F(1,105)=5.08$, $\left.p=.03, \eta_{G}^{2}=.01\right)$.

Both men and women were very likely to choose the attractive-and-highincome profiles for long-term and short-term choices with the Alternative set, with all choice proportions above $87 \%$ (see Table 2). They were more likely to choose the attractive-and-high-income profiles for long-term partners than for short-term partners 
$\left(F(1,105)=40.78, p<.001, \eta_{G}^{2}=.08\right)$. Again, this difference between short- and longterm partners was more pronounced for women than for men $(F(1,105)=6.05, p=.02$, $\left.\eta_{G}^{2}=.01\right)$. Overall, the aggregate choice proportions are highly consistent with previous findings on the sex differences in mate preferences and the moderation effect of shortterm (vs long-term) mating contexts (Buss, 1989; Feingold, 1990; 1992; Kenrick et al., 1993; Li \& Kenrick, 2006; Todd et al., 2007).

\section{Table 2}

Percentage of the more attractive profile being chosen in pairwise choices (standard error in parentheses).

\begin{tabular}{llcc}
\hline \multirow{2}{*}{ Mating context } & Stimulus set & \multicolumn{2}{c}{ Attractive chosen } \\
\cline { 3 - 4 } & & Men & Women \\
\hline Short-term & Main & $0.72(0.03)$ & $0.60(0.03)$ \\
Long-term & Main & $0.57(0.04)$ & $0.35(0.03)$ \\
Short-term & Alternative & $0.89(0.01)$ & $0.87(0.02)$ \\
Long-term & Alternative & $0.92(0.01)$ & $0.95(0.01)$ \\
\hline
\end{tabular}

Note that our participants sometimes chose the profile that was both less attractive and had less income in the Alternative set. Some of these choices may be careless errors. However, it is also possible that people might not prefer high income to low income. People may even prefer low income to high income, especially in short-term partner selection. Such preference patterns would make people more likely to choose the less attractive profile with less income. 


\section{Qualitative model evaluation}

Are participants' choices consistent with the transitive or LS models? We examined whether participants' choice frequencies were consistent with the models' predictions. Figure 1 shows the percentage of participants whose choice frequencies were consistent with the models' predictions in each condition respectively. With the Main set, individual participants' choice frequencies are largely consistent with transitive models' predictions, especially WST, MST, and MMTP, with all the consistencies above $72 \%$. SST scored slightly lower, between $36 \%$ and $51 \%$, depending on the experimental conditions. Yet, these numbers were much higher than the counterparts of the LS models.

The Alternative set showed slightly different patterns. A large proportion of participants' choices were consistent with the transitive models. Many participants' choices were seemingly consistent with the LS models too. This was likely due to the fact that the Alternative sets were non-diagnostic for most of the participants. As shown in Figure 1, seventy-one percent of the data that were consistent with the seven standard models were also consistent with the corresponding constrained models (i.e., the brown bars). This number rose to $89 \%$ when women selected long-term partners. These patterns suggested that most participants preferred high income to low income in partner selection, especially when women were making long-term partner selection. Still, a significant minority of participants did not follow the constrained models, among whom the majority were consistent with the transitive models whereas very few were consistent with the LS models. 


\section{Figure 1}

Qualitative consistency between participants' choice frequencies and model

predictions. The whole bar heights represent the overall consistency percentage for the standard models. The brown parts represent the constrained models and the green parts represent otherwise.

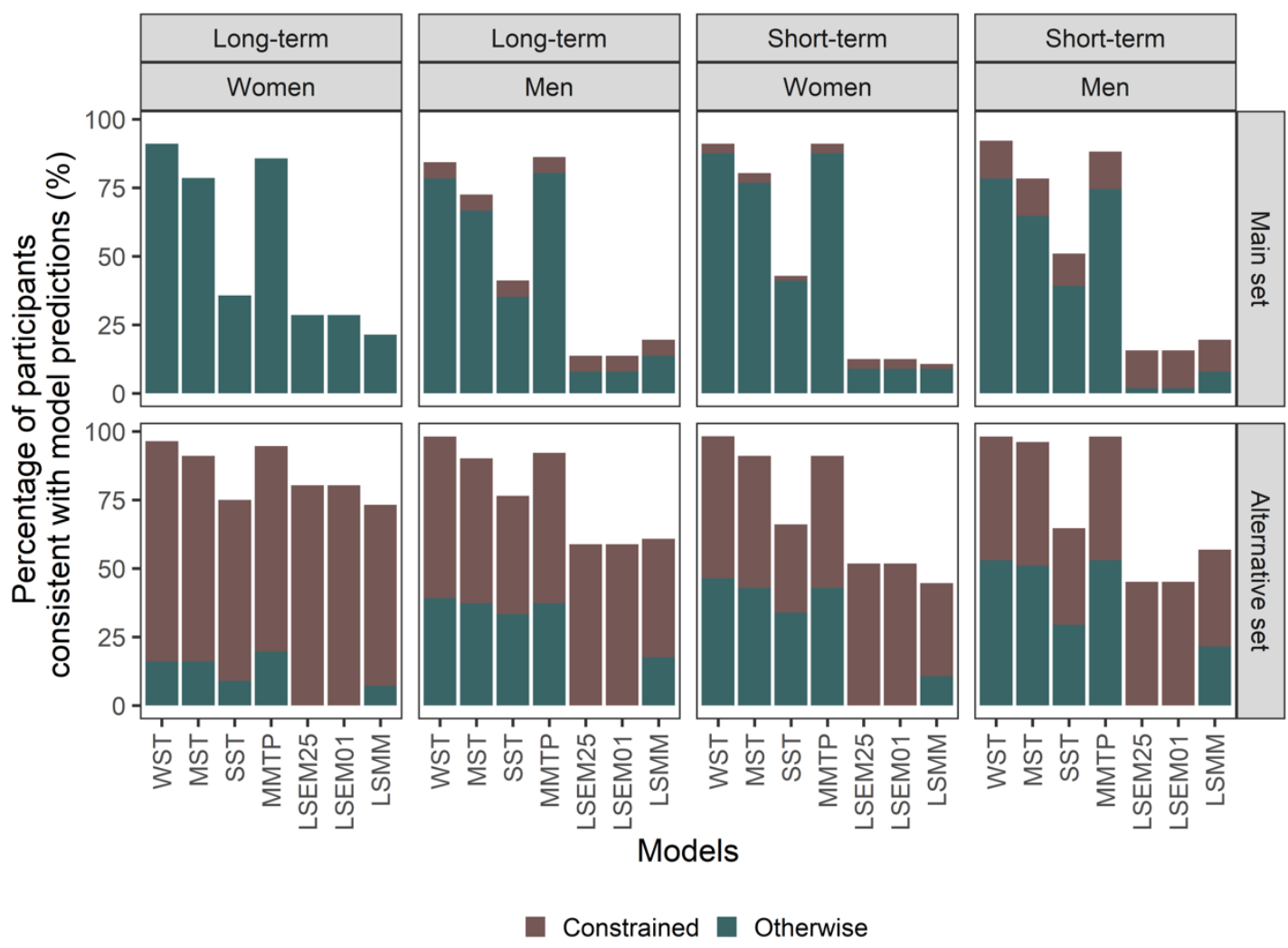

On the individual level, the vast majority of participants were consistent with at least one of the four transitive models. Only seven participants (three women and four men) were inconsistent with the transitive models. Interestingly, neither were they consistent with the LS models, suggesting that their data deviated from all of the substantive models considered in this paper. 
Overall, our preliminary assessment of individual-level choice frequencies in relation to the various models' predictions suggested that participants' choices were mostly consistent with transitive models but less likely to be consistent with the LS models, and that most participants' choices were consistent with the constrained models (i.e., the preference order of $A>B>C>D>E$ ) in the Alternative set.

\section{Quantitative model comparisons}

\section{Overall model performance}

We next examined the transitivity of mate preferences by comparing the standard transitive models against the standard models that allowed for intransitive preferences using the Bayesian method. Here, we combined the Main and Alternative sets for each participant in each condition. For each model, we obtained an aggregate $\log$ Bayes factor (BF) against the saturated model by summing up the log Bayes factors across participants. We did this separately for different sexes and different mating conditions as in Table 3. Positive values mean that the model outperformed the saturated model whereas negative values mean that the model performed worse than the saturated model. In aggregate, all transitive models outperformed the saturated model with decisive aggregate Bayes factors. The strong stochastic transitivity (SST), in particular, stood out as the best-performing model for both women and men, in both long-term and short-term mating contexts. By contrast, the lexicographic semiorder models rarely outperformed the saturated model, suggesting that our participants' choices were unlikely to follow the lexicographic rules. The only exception was LSEM01, which had an aggregate log BF of 9 for women making 
long-term partner choice, outperforming the saturated model. Yet LSEM01 lagged far behind all transitive models.

\section{Table 3}

Bayesian model selection results with all data. Aggregate log Bayes factors (BF) against the saturated model (using natural base) are in the left column. Positive values mean that the model outperformed the saturated model whereas negative values mean that the model performed worse than the saturated model. Percentages of best individual-level fits are in the right column.

\begin{tabular}{|c|c|c|c|c|c|c|c|c|}
\hline \multirow{3}{*}{ Model } & \multicolumn{4}{|c|}{ Aggregate $\log \mathrm{BF}$} & \multicolumn{4}{|c|}{$\%$ Best $\log \mathrm{BF}$} \\
\hline & \multicolumn{2}{|c|}{ Long-term } & \multicolumn{2}{|c|}{ Short-term } & \multicolumn{2}{|c|}{ Long-term } & \multicolumn{2}{|c|}{ Short-term } \\
\hline & Women & Men & Women & Men & Women & Men & Women & Men \\
\hline WST & 230 & 203 & 231 & 212 & 0 & 0 & 0.04 & 0 \\
\hline MST & 334 & 304 & 356 & 324 & 0.23 & 0.22 & 0.13 & 0.22 \\
\hline SST & 344 & 317 & 377 & 335 & 0.46 & 0.61 & 0.73 & 0.71 \\
\hline MMTP & 187 & 170 & 201 & 178 & 0.02 & 0 & 0.02 & 0 \\
\hline LSEM01 & 9 & -76 & -258 & -211 & 0.29 & 0.14 & 0.09 & 0.08 \\
\hline LSEM25 & -45 & -125 & -305 & -255 & 0 & 0.02 & 0 & 0 \\
\hline LSMM & -146 & -183 & -223 & -193 & 0 & 0.02 & 0 & 0 \\
\hline
\end{tabular}

Note. The LS models provided extremely bad fits to a few participants and the numerator in Equation 1 became zero in our Monte Carlo simulation (resulting in a BF of zero). In such cases, we increased the numerator in Equation (1) to 1e-7 
(because the simulation sample size is 1e7) to facilitate the calculation of the aggregate $\log \mathrm{BF}$ in Table 3. No modification was made to the $\log \mathrm{BF}$ values of transitive models.

Transitive models also provided the best log BF (against the saturated model) to the vast majority of our participants. Overall, $69 \%$ women and $83 \%$ men were best described by the transitive models in long-term choice and $89 \%$ women and $93 \%$ men in short-term choice (see Table 3 for a detailed breakdown). Notably, SST, the most restrictive transitive model, provided the best fit to $46 \%$ women and $61 \%$ men in long-term choice and $73 \%$ women and $71 \%$ men in short-term choice. MST also obtained the best fit to a significant minority of participants. By contrast, lexicographic semiorder models only provided the best fit to a small fraction of participants. LSEM01 best fit 29\% women and 14\% men in long-term choice and $9 \%$ women and $8 \%$ men in short-term choice. LSEM25 and LSMM provided the best fit to only one dataset respectively. The saturated model provided no best fit at all.

\section{Individual-level pairwise comparisons}

The individual-level pairwise Bayes factors between standard transitive and standard intransitive models provide direct evidence for transitive preferences. By convention, a Bayes factor greater than 3 was considered positive evidence for the winning model (Kass \& Raftery, 1995; Raftery, 1996). In the comparison between transitive models and the saturated model (see the top row of Figure 2 and Table 4), almost all individual-level Bayes factors were greater than 3, supporting the transitive 
models for both men and women, in both long-term and short-term mating contexts. A majority of the Bayes factors were larger than 10, providing substantial evidence for the transitive models against the saturated model.

The pairwise comparisons between the transitive and LS models also suggested that most participants' choices were better described by transitive models $\left(2^{\text {nd }}\right.$ to $4^{\text {th }}$ rows, Figure 2 ). As summarized in Table 4 , a majority of the pairwise Bayes factors (ranging from $57 \%$ to $100 \%$, depending on the models and conditions) were greater than 3. By contrast, very few participants provided non-anecdotal evidence for LS models against the transitive models with Bayes factors smaller than 1/3. This was the case for the two lexicographic semiorder error models (i.e. LSEM01 and LSEM25). There was almost no participant who provided non-anecdotal evidence for the lexicographic semiorder mixture model against transitive models.

\section{Figure 2}

Violin plots of the Bayes factors between transitive models and models that allow for intransitive preferences. Values greater than 1 support a transitive over an intransitive model and values smaller than 1 support an intransitive model over a transitive one. The dashed lines indicate Bayes factor of 3 or 1/3 respectively. 


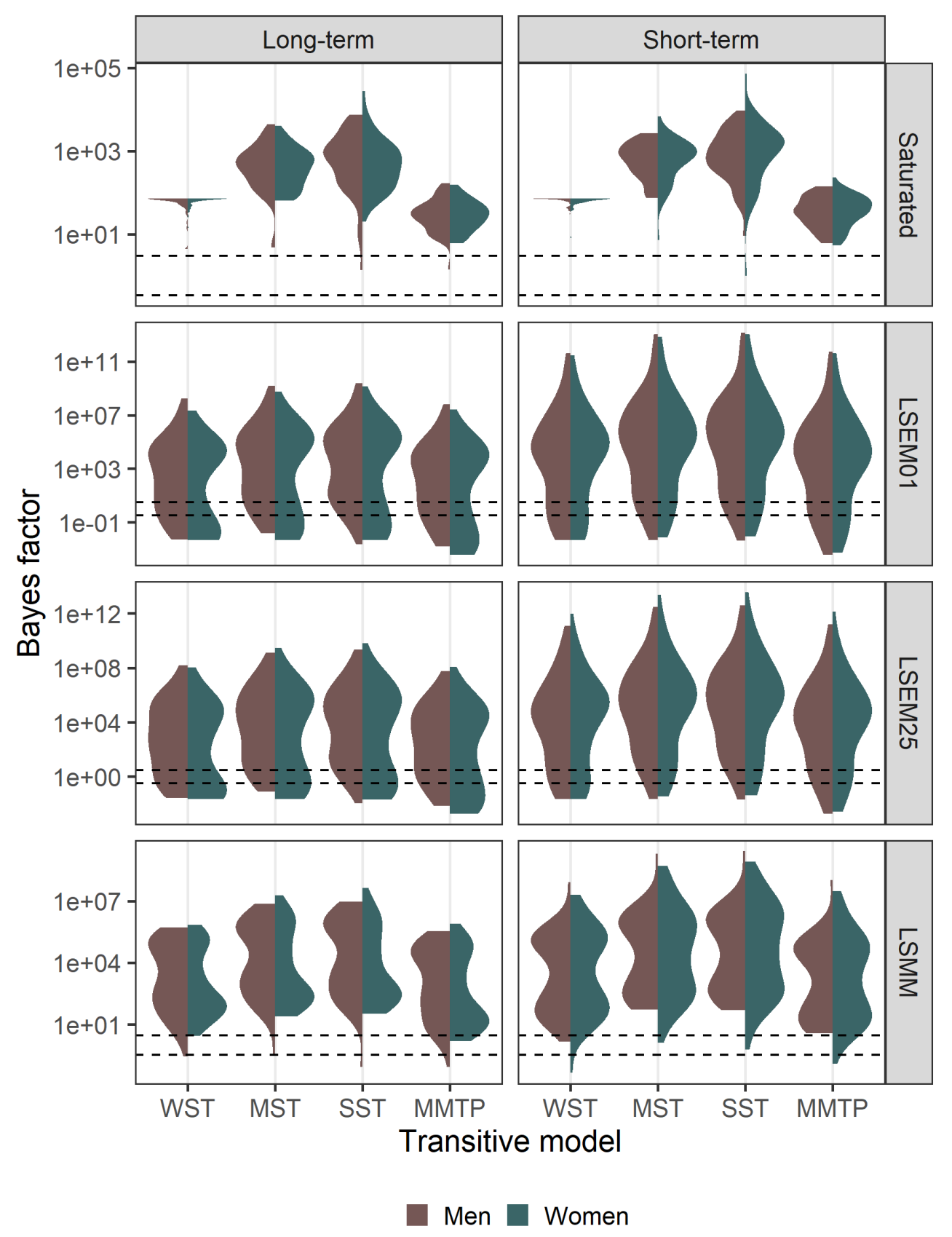

Table 4

Proportions of pairwise Bayes factors that offer non-anecdotal evidence for the winning model (i.e., Bayes factors are larger than 3 or smaller than 1/3). The values outside of the parenthesis indicate the proportions of Bayes factors that favor the transitive models (i.e. the column model) while the values inside the parenthesis 
indicate the proportions of Bayes factors that favor the models that allow for intransitive preferences (i.e. the row model). All models in this table were standard models.

\begin{tabular}{|c|c|c|c|c|c|c|c|c|}
\hline \multicolumn{9}{|c|}{ Long-term } \\
\hline & \multicolumn{4}{|c|}{ Women } & \multicolumn{4}{|c|}{ Men } \\
\hline & WST & MST & SST & MMTP & WST & MST & SST & MMTP \\
\hline Saturated & $\begin{array}{c}1.00 \\
(0.00)\end{array}$ & $\begin{array}{c}1.00 \\
(0.00)\end{array}$ & $\begin{array}{c}1.00 \\
(0.00)\end{array}$ & $\begin{array}{c}1.00 \\
(0.00)\end{array}$ & $\begin{array}{c}1.00 \\
(0.00)\end{array}$ & $\begin{array}{c}1.00 \\
(0.00)\end{array}$ & $\begin{array}{c}0.98 \\
(0.00)\end{array}$ & $\begin{array}{c}0.98 \\
(0.00)\end{array}$ \\
\hline LSEM01 & $\begin{array}{c}0.61 \\
(0.38)\end{array}$ & $\begin{array}{c}0.64 \\
(0.27)\end{array}$ & $\begin{array}{c}0.66 \\
(0.29)\end{array}$ & $\begin{array}{c}0.57 \\
(0.38)\end{array}$ & $\begin{array}{c}0.71 \\
(0.18)\end{array}$ & $\begin{array}{c}0.78 \\
(0.10)\end{array}$ & $\begin{array}{c}0.82 \\
(0.08)\end{array}$ & $\begin{array}{c}0.67 \\
(0.20)\end{array}$ \\
\hline LSEM25 & $\begin{array}{c}0.57 \\
(0.27)\end{array}$ & $\begin{array}{c}0.68 \\
(0.21)\end{array}$ & $\begin{array}{c}0.66 \\
(0.20)\end{array}$ & $\begin{array}{c}0.59 \\
(0.29)\end{array}$ & $\begin{array}{c}0.76 \\
(0.16)\end{array}$ & $\begin{array}{c}0.84 \\
(0.10)\end{array}$ & $\begin{array}{c}0.84 \\
(0.04)\end{array}$ & $\begin{array}{c}0.75 \\
(0.16)\end{array}$ \\
\hline LSMM & $\begin{array}{c}0.96 \\
(0.00) \\
\end{array}$ & $\begin{array}{c}1.00 \\
(0.00) \\
\end{array}$ & $\begin{array}{c}1.00 \\
(0.00) \\
\end{array}$ & $\begin{array}{c}0.96 \\
(0.00) \\
\end{array}$ & $\begin{array}{c}0.98 \\
(0.02) \\
\end{array}$ & $\begin{array}{c}0.98 \\
(0.02) \\
\end{array}$ & $\begin{array}{c}0.98 \\
(0.02) \\
\end{array}$ & $\begin{array}{c}0.92 \\
(0.02) \\
\end{array}$ \\
\hline \multicolumn{9}{|c|}{ Short-term } \\
\hline & \multicolumn{4}{|c|}{ Women } & \multicolumn{4}{|c|}{ Men } \\
\hline & WST & MST & SST & MMTP & WST & MST & SST & MMTP \\
\hline Saturated & $\begin{array}{c}1.00 \\
(0.00)\end{array}$ & $\begin{array}{c}1.00 \\
(0.00)\end{array}$ & $\begin{array}{c}0.98 \\
(0.00)\end{array}$ & $\begin{array}{c}1.00 \\
(0.00)\end{array}$ & $\begin{array}{c}1.00 \\
(0.00)\end{array}$ & $\begin{array}{c}1.00 \\
(0.00)\end{array}$ & $\begin{array}{c}1.00 \\
(0.00)\end{array}$ & $\begin{array}{c}1.00 \\
(0.00)\end{array}$ \\
\hline LSEM01 & $\begin{array}{c}0.79 \\
(0.16)\end{array}$ & $\begin{array}{c}0.86 \\
(0.07)\end{array}$ & $\begin{array}{c}0.86 \\
(0.07)\end{array}$ & $\begin{array}{c}0.77 \\
(0.14)\end{array}$ & $\begin{array}{c}0.78 \\
(0.16)\end{array}$ & $\begin{array}{c}0.86 \\
(0.08)\end{array}$ & $\begin{array}{c}0.86 \\
(0.06)\end{array}$ & $\begin{array}{c}0.76 \\
(0.18)\end{array}$ \\
\hline LSEM25 & $\begin{array}{c}0.79 \\
(0.14)\end{array}$ & $\begin{array}{c}0.89 \\
(0.05)\end{array}$ & $\begin{array}{c}0.91 \\
(0.09)\end{array}$ & $\begin{array}{c}0.79 \\
(0.12)\end{array}$ & $\begin{array}{c}0.82 \\
(0.10)\end{array}$ & $\begin{array}{c}0.92 \\
(0.06)\end{array}$ & $\begin{array}{c}0.92 \\
(0.04)\end{array}$ & $\begin{array}{c}0.82 \\
(0.12)\end{array}$ \\
\hline LSMM & $\begin{array}{c}0.98 \\
(0.02)\end{array}$ & $\begin{array}{c}0.98 \\
(0.00)\end{array}$ & $\begin{array}{c}0.98 \\
(0.00)\end{array}$ & $\begin{array}{c}0.96 \\
(0.02)\end{array}$ & $\begin{array}{c}0.98 \\
(0.00)\end{array}$ & $\begin{array}{c}1.00 \\
(0.00)\end{array}$ & $\begin{array}{c}1.00 \\
(0.00)\end{array}$ & $\begin{array}{c}1.00 \\
(0.00)\end{array}$ \\
\hline
\end{tabular}

Nonetheless, a few participants were best fit by the lexicographic semiorder models. Some datasets even provided non-anecdotal evidence for the LS models (mostly LSEM01) against the transitive models with Bayes factors greater than 3 (see Figure 2 and Table 4). But can we conclude that these participants displayed intransitive mate preferences? To answer this question, we combined the quantitative fits with the qualitative model consistencies on the individual level. We checked whether the participants who were best fit by the LS models displayed obvious 
qualitative violations of transitive models. After close inspection, we found that across mating contexts and sexes, 31 of the 35 datasets best described by the LS models were qualitatively consistent with at least one of the transitive models (see the second columns of each of the four contingency tables in Table 5). Thus, we could not conclude that those participants were using a lexicographic choice strategy. Rather, LS models outperformed transitive models, such as WST or MMTP, likely because the former was more parsimonious than the latter.

Among the seven participants whose data were qualitatively inconsistent with any of the transitive models, they were qualitatively inconsistent with the LS models either. Thus it was unclear whether they were following lexicographic rules. Quantitatively, 3 of them were best fit by MST, 1 by MMTP, 2 by LSEM01, and 1 by LSMM. None of them deviated from these models substantially enough to support the saturated model. Those data offered some evidence of intransitive preferences from a very small fraction of participants, although none of them unambiguously supported the LS models that allowed for intransitive preferences.

By closely associating quantitative model fits with qualitative patterns, we found substantial evidence supporting the transitive models against the LS models in repeated mate choice data. The results also suggested that the saturated model was overly flexible and could not outperform other models with model complexity penalized, even when the choice data were qualitatively consistent with none of the substantive models. 


\section{Table 5}

Contingency tables between qualitative consistency with the transitive models and quantitative fit by the transitive models for women and men in long-term and shortterm mating contexts respectively. indicating the number of participants whose data were or were not qualitatively consistent with the transitive models and the number of participants whose data were or were not quantitatively best fit by the transitive models.

\begin{tabular}{|c|c|c|c|c|c|c|c|}
\hline \multicolumn{8}{|c|}{ Long-term } \\
\hline \multicolumn{4}{|c|}{ Women $(N=56)$} & \multicolumn{4}{|c|}{$\operatorname{Men}(N=51)$} \\
\hline \multirow{4}{*}{ Qualitative } & & \multicolumn{2}{|c|}{ Quantitative } & \multirow{4}{*}{ Qualitative } & \multirow{4}{*}{$\begin{array}{l}\text { Yes } \\
\text { No }\end{array}$} & \multicolumn{2}{|c|}{ Quantitative } \\
\hline & & Yes & No & & & Yes & No \\
\hline & Yes & 39 & 15 & & & 41 & 7 \\
\hline & No & 1 & 1 & & & 1 & 2 \\
\hline \multicolumn{8}{|c|}{ Short-term } \\
\hline \multicolumn{4}{|c|}{ Women $(N=56)$} & \multicolumn{4}{|c|}{$\operatorname{Men}(N=51)$} \\
\hline \multirow{4}{*}{ Qualitative } & & \multicolumn{2}{|c|}{ Quantitative } & & & \multicolumn{2}{|c|}{ Quantitative } \\
\hline & & Yes & No & & & Yes & No \\
\hline & Yes & 49 & 6 & \multirow{2}{*}{ Qualitative } & Yes & 46 & 4 \\
\hline & No & 1 & $\mathbf{0}$ & & No & 1 & $\mathbf{0}$ \\
\hline
\end{tabular}

Note. In each of the contingency tables, "Qualitative" rows indicate the numbers of participants whose data were or were not qualitatively consistent with the transitive models. "Quantitative" columns indicate the number of participants whose data were or were not quantitatively best fit by the transitive models.

\section{Model space}

The transitive and LS models have more nuanced differences beyond the transitive versus intransitive distinction. We further analyzed their relationships by measuring the pairwise distances and mapping them onto one single space via 
multidimensional scaling (see Broomell et al., 2011 and He et al., 2020 for similar approaches to comparing economic decision models). The pairwise model distances were calculated as the Euclidean distances in the 233-dimensional space of each model $M$ 's $\log$ BF against the saturated model $S$, i.e. $\log B F_{M, S}$, across participants. As in Figure 3, the transitive models were located together and were distant from the LS models. The two lexicographic semiorder error models (LSEM01 and LSEM25) were located close to each other but were distant from the lexicographic semiorder mixture model (LSMM). This was consistent with the extensive evidence suggesting that stochastic specifications played an unneglectable role in model predictions (Loomes \& Sudgen, 1995; Regenwetter et al., 2011; Bhatia \& Loomes, 2017; He et al., 2019). The saturated model lay between the transitive models and the lexicographic semiorder models, as it permitted intransitive preferences but also subsumed the transitive models as special cases.

\section{Figure 3}

Quantitative model space via classical multidimensional scaling. 


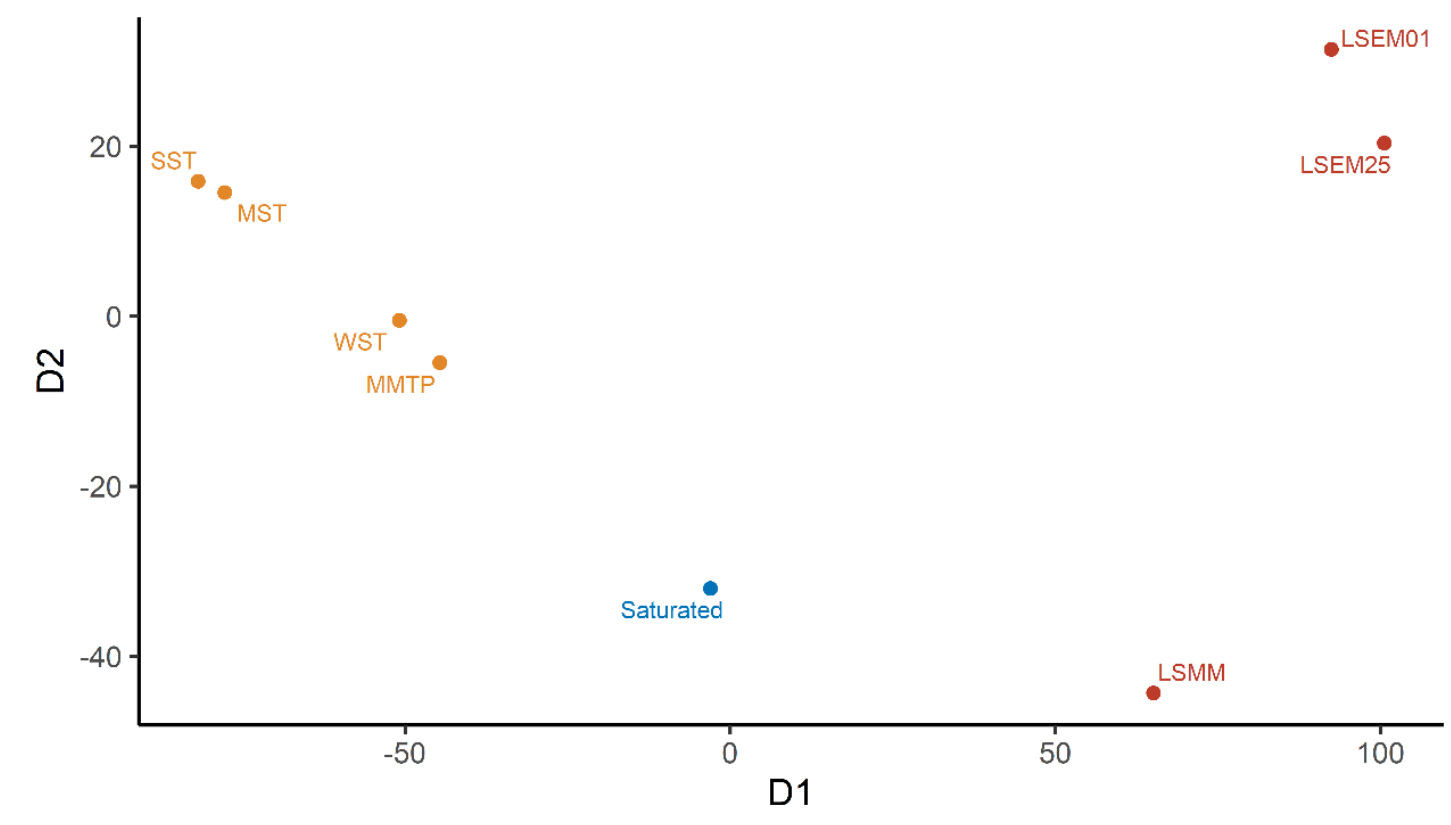

- LS - Saturated - Transitive

\section{Clusters of mate preferences}

On the individual level, participants had heterogeneous weighting of different attributes. We mapped out these heterogeneous preferences by applying $k$-medoids cluster analysis (also known as partitioning around medoids) to their choice probabilities (see Hatz et al., 2020 for a similar approach). The two-cluster solution provided the best fit with the Silhouette width of 0.18 . Figure 4 displays the two clusters mapped onto the first two principal components of the data space. Here, each point represents one participant. The two clusters roughly corresponded to different weights assigned to the two attributes. The left-hand-side (LHS) cluster more heavily weighted annual income, selecting the high-income profile more often than the attractive profile in the Main set. Figure 5 shows the average choice proportions for the two clusters. Each point represents the proportion of the more attractive option 
being chosen in the pairwise choice, ranging from $0 \%$ (the plot center) and $100 \%$ (the white circle). For example, with the Main set, the RHS-cluster participants chose A (i.e., the more attractive option) $67 \%$ of the time in the pairwise choices between $A$ and $B$ whereas the participants in the LHS cluster only chose $A 21 \%$ of the time in the long-term context. The same pattern also emerged from other choice pairs. With the Alternative set, the distinction was much smaller since the two attributes favored the same option in this set for the majority of our participants.

\section{Figure 4}

Clusters of mate preferences mapped on the first two principal components. The first two components explain $31.8 \%$ of the variance of the original 40-dimensional data.

Cluster affiliation is indicated by colors along with the 95\%-level ellipse. LHS = Lefthand side; $R H S=$ Right-hand side .

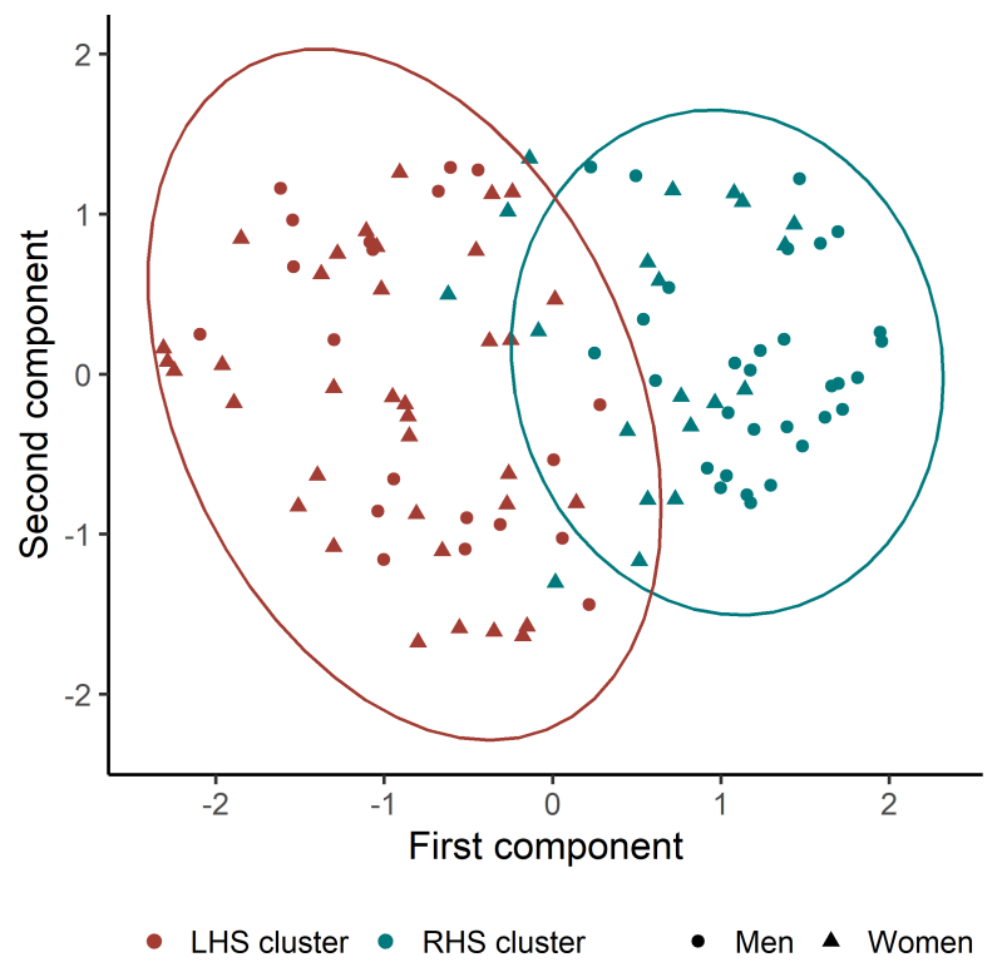




\section{Figure 5}

Average choice proportions in the two clusters. The plot center is $0 \%$ and the white circle is $100 \%$ of choosing the more attractive option in each choice pair.

\section{Main set}
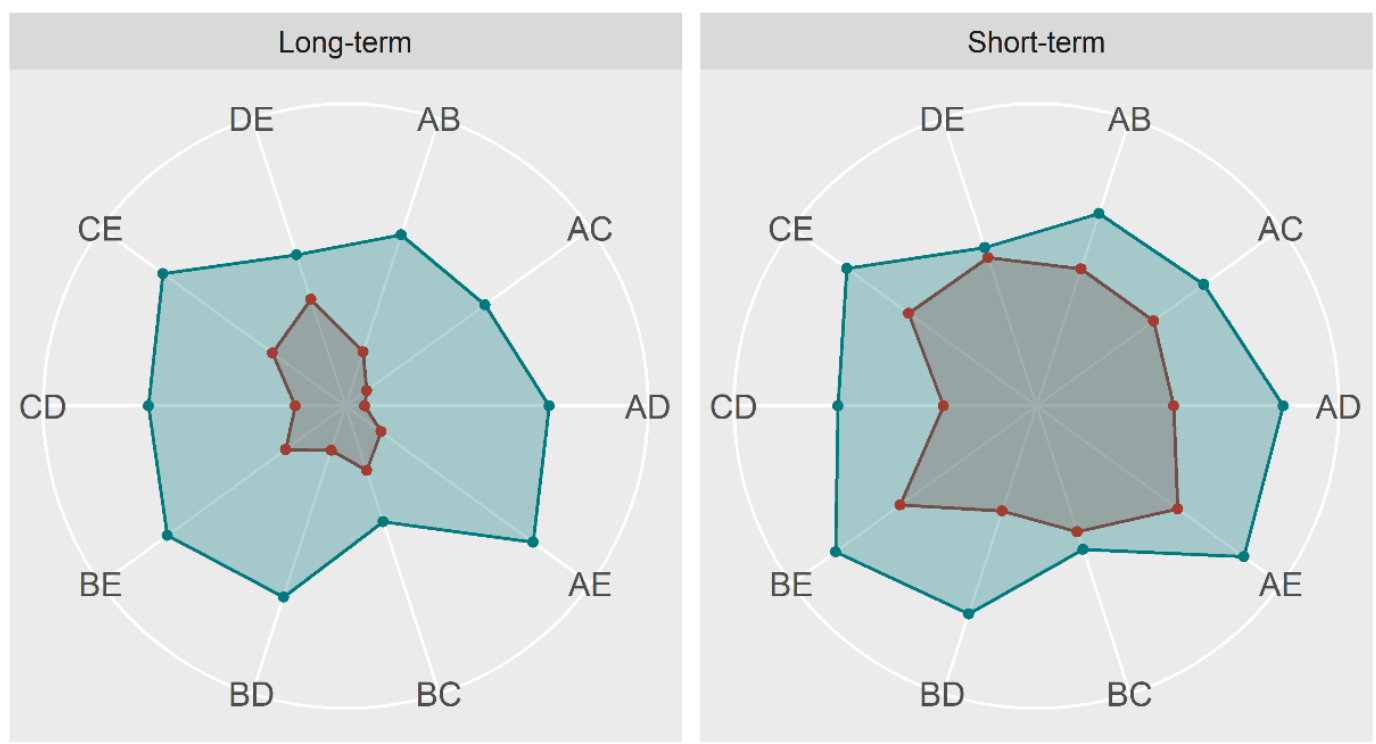

\section{Alternative set}
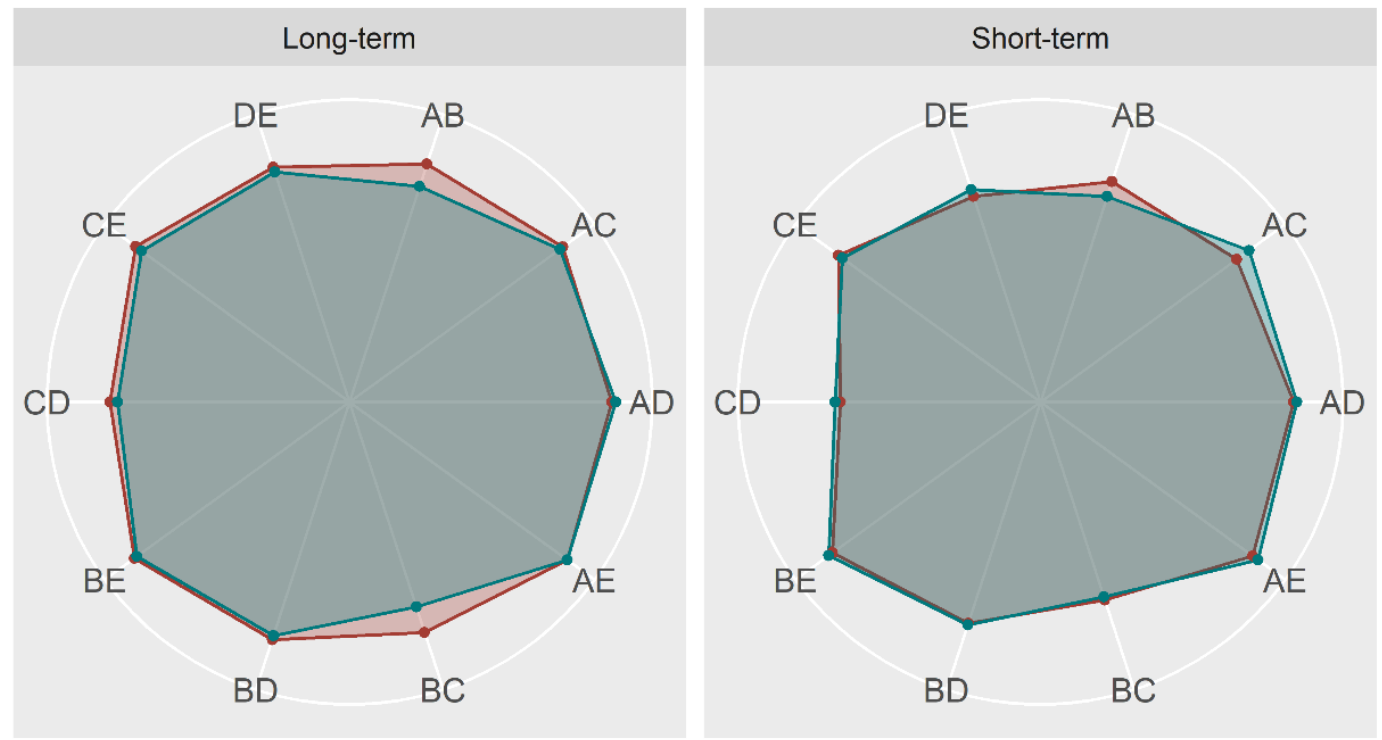

- LHS cluster 
A majority of woman participants (64\%) were assigned to the LHS cluster. By contrast, the right-hand-side (RHS) cluster tended to more heavily weight attractiveness, selecting the attractive option more often than the high-income option. A majority of man participants (62\%) were assigned to the RHS cluster.

\section{Robustness check with data filtering}

One potential issue with the Bayesian model comparisons was that nondiagnostic designs (e.g., the Alternative set for many participants) may have inflated the goodness of fit of the transitive models and favor the transitive models against the LS models in Bayesian model selection. Those non-diagnostic design sets were unable to uncover the intransitive patterns even if intransitive patterns could potentially show up. To test this possibility, we ran a robustness check by excluding the non-diagnostic datasets (i.e. the data that can be better accounted for by the constrained models, rather than the standard models in Table 1). Specifically, we identified the diagnostic data by running a Bayesian model comparison of 15 models (including 8 standard models and 7 constrained models) at the individual-level. The complete model comparison revealed whether a dataset was best accounted for by the constrained models.

\section{Figure 6}

Percentage of best log BF against the saturated model across participants in each condition using pairwise Bayes factor $=1$ as the cutoff point. The numeric percentage above each bar is the percentage of the standard models (i.e., the green bars). The 
datasets best fit by a constrained model (i.e., the brown bars) were considered nondiagnostic for the transitivity test and were thus removed from subsequent transitivity testing.

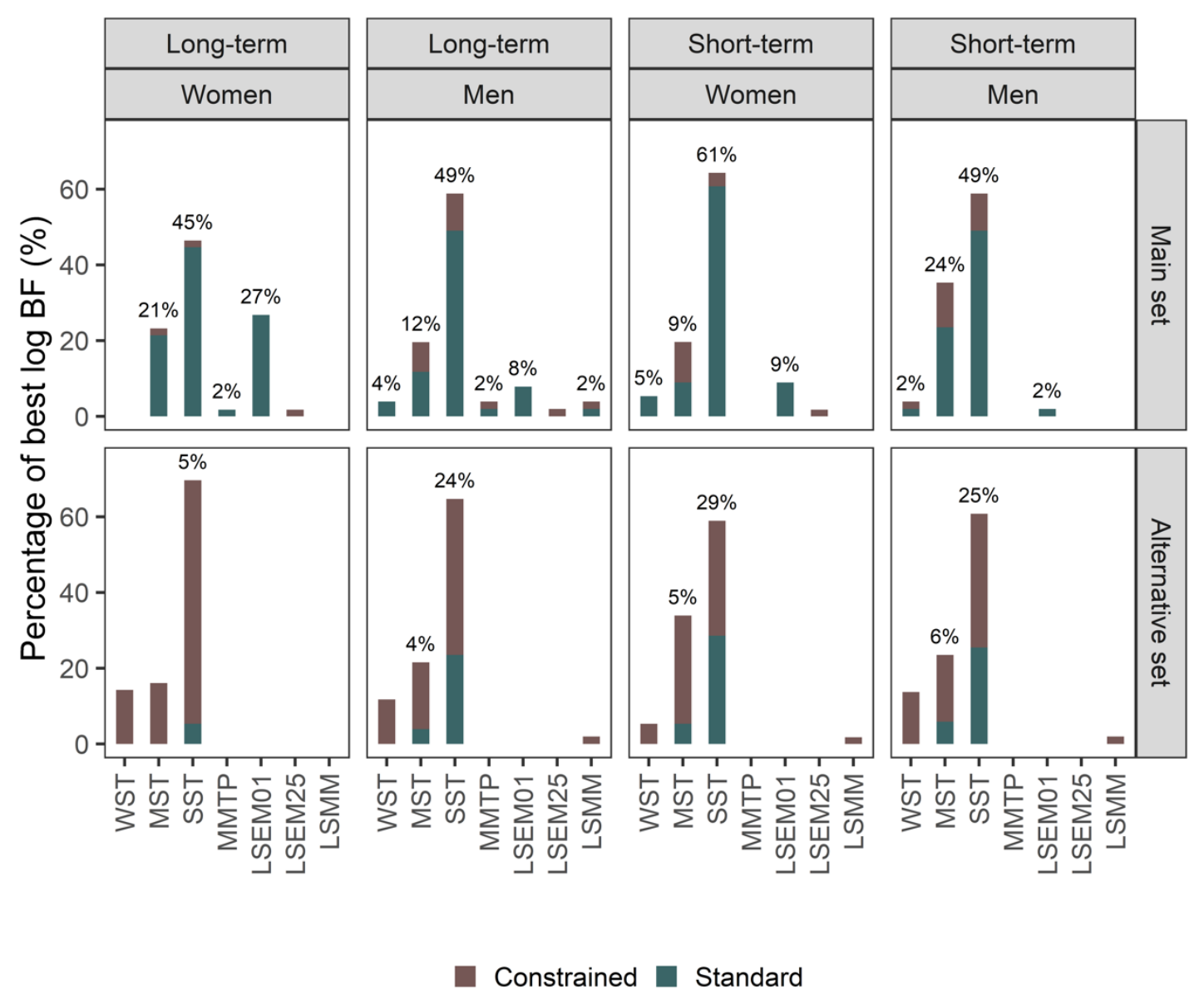

As shown in Figure 6, approximately $17 \%$ of the datasets in the Main set across conditions were best described by the constrained models, whereas $76 \%$ in the Alternative set were best described by the constrained models. This was consistent with the fact that most participants preferred high income to low income in mate preferences. It was also consistent with the results in Figure 1 that a majority of data with the Alternative set were qualitatively consistent with the constrained models. Those data that were best fit by the constrained models were considered non- 
diagnostic for transitivity testing and thus removed from the subsequent robustness check. By doing so, we were able to identify the suitable design set for transitivity testing for each participant in each condition.

\section{Table 6}

Bayesian model selection results with diagnostic data only. Aggregate log Bayes factors (BF) against the saturated model (using natural base) and the percentage of best $B F$.

\begin{tabular}{|c|c|c|c|c|c|c|c|c|}
\hline \multirow{3}{*}{ Model } & \multicolumn{4}{|c|}{ Aggregate $\log \mathrm{BF}$} & \multicolumn{4}{|c|}{$\%$ Best $\log \mathrm{BF}$} \\
\hline & \multicolumn{2}{|c|}{ Long-term } & \multicolumn{2}{|c|}{ Short-term } & \multicolumn{2}{|c|}{ Long-term } & \multicolumn{2}{|c|}{ Short-term } \\
\hline & Women & Men & Women & Men & Women & Men & Women & Men \\
\hline WST & 114 & 105 & 138 & 115 & 0 & 0.04 & 0.05 & 0.02 \\
\hline MST & 176 & 164 & 217 & 177 & 0.23 & 0.15 & 0.12 & 0.27 \\
\hline SST & 179 & 167 & 233 & 180 & 0.48 & 0.70 & 0.76 & 0.69 \\
\hline MMTP & 103 & 93 & 122 & 98 & 0.02 & 0.02 & 0 & 0 \\
\hline LSEM01 & -221 & -262 & -417 & -376 & 0.27 & 0.08 & 0.08 & 0.02 \\
\hline LSEM25 & -234 & -277 & -435 & -384 & 0 & 0.02 & 0 & 0 \\
\hline LSMM & -151 & -194 & -239 & -213 & 0 & 0.04 & 0.05 & 0.02 \\
\hline
\end{tabular}

The results with the diagnostic data are summarized in Table 6. In aggregate, SST provided the best aggregate log BF (against the saturated model) across participants in all conditions, followed by MST, WST and MMTP. All transitive 
models outperformed all LS models in this regard. As can be seen in Figure 6, transitive models, especially SST and MST provided the best log BF to the vast majority of our participants when only the diagnostic data were considered. The only LS model that provided a significant proportion of best log BF is LSEM01, which for example best accounted for $27 \%$ of the data when women making long-term choices. Overall, our key findings with the full set of data were replicated with the diagnostic subsets.

\section{Discussion}

It is widely held that human mate preferences are rational in a way to maximize their reproductive success, financial benefits, and social credit (Buss \& Schmitt, 1993; Eagly \& Wood, 1999; Kenrick et al., 1993). However, systematic testing of mate choice rationality from the decision-theoretic perspective is lacking. In this paper, we presented a systematic experimental test of rationality in human mate preferences through the lens of transitivity, a fundamental hallmark of rational decision-making. Our results suggested that most participants' mate preferences were transitive for both long-term and short-term mate choices, validating the assumption of rationality underlying the influential theoretical accounts of mate preferences. Our work complements the extensive research on the psychological, social, and evolutionary determinants of mate preferences and opens up new directions for investigating human mating behavior. 


\section{Transitive vs LS models}

Our major contribution is in presenting a systematic test of transitive mate preferences, against the class of lexicographic semiorder models that allow for intransitive preferences. The decision-theoretic approach is uniquely valuable to the study of mate choice rationality, by subjecting the latter to experimental testing. The overall evidence for transitive mate preferences is compelling. A majority of participants' data were best explained by strong stochastic transitivity, the most parsimonious transitive model, in Bayesian model selection. Transitive mate preferences are consistent with the proposals that human mating strategy may be acquired through sexual selection, a process of optimizing individual survival and reproductive success (Buss \& Schmitt, 1993; Gangestad \& Simpson, 2000). In contrast, few participants showed choice patterns that were consistent with LS models.

Our results were consistent with some related studies. A few animal studies suggest that their mate preferences are mostly transitive (e.g., Arbuthnott et al., 2017; Dechaume-Moncharmont et al., 2013). A recent study on human mating strategies by Brandner et al. (2020) suggests that compensatory strategies describe choice data more accurately than non-compensatory strategies, which also imply transitive mate choice. Hatz et al. (2020) evaluated the transitivity of risky sexual decision-making. They formulated risky sexual decision making using physical attractiveness (the same way as we formulate the mate choice) and the probability of contracting a sexually transmitted infection. Likewise, they found that their participants' risky sexual 
decision-making was more consistent with a transitive model called Weak Order Mixture Model (Regenwetter \& Davis-Stober, 2012) than the lexicographic semiorder mixture models. The converging evidence suggests that the test of transitivity is a reliable approach to validating the rationality of mate preferences in humans and animals.

We extended previous studies by considering a wide range of models and stimuli. Hatz et al. (2020) used a similar design to test the transitivity of sexual decision making, contrasted the Weak Order Mixture Model (Regenwetter \& DavisStober, 2012) as a stochastic model of transitivity to the intransitive competitor model of Davis-Stober (2012). As transitivity testing can be sensitive to the different stochastic assumptions, we involved four prominent transitive models that make different stochastic assumptions. We also involved four prominent intransitive models, including the saturated model and three variants of lexicographic semiorder model (Tversky, 1969). We also created stimuli adaptively for each participant, contingent on their attractiveness ranking for each image set, in different mating contexts. These models and designs expanded the scope of our transitivity testing.

Of course, there were exceptions. In our experiment, seven participants' data were qualitatively inconsistent with the transitive models. From the normative standard of rationality, these participants were less rational in mate choice. However, it has also been argued that the use of simple heuristics can be ecologically rational, even if they sometimes violate traditional standards of rationality such as transitivity (see Todd et al., 2012 for thorough discussions). From a cognitive perspective, 
transitive strategies typically require loads of cognitive resources for rigorous calculation whereas simple heuristic strategies were less cognitively costly. Thus, it is possible the simpler intransitive strategies can be the consequence of rational use of the limited cognitive resources (Payne et al., 1993; Lieder \& Griffiths, 2017).

\section{Ecologically and socially rational mate preferences}

Rational mate choice, of course, can be evaluated from other perspectives. It is well known that men tend to assign more weight to physical attractiveness whereas women tend to place more weight on financial resources (Buss, 1989; Buss \& Barnes, 1986; Buss et al., 2001; Feingold, 1990; 1992). Our aggregate results and clustering analysis reveal similar patterns: Most men tended to select the attractive candidate while most women tended to select the higher-income candidate. Of course, this sex difference is not an ideal causal inference in the experiment as men and women dealt with different sets of stimuli. However, this finding is consistent with a large body of existing research on sex differences in partner selection.

This sex difference may be ecologically rational when we consider our evolutionary roots and social contexts. From an evolutionary perspective, our goals are successful survival, reproduction, and raising offspring. Physical attractiveness is believed to be a signal of high fertility in women and, thus, men's emphasis on physical attractiveness can be an adaptive strategy for maximizing reproductive success (Symons, 1979). In contrast, women's emphasis on financial resources can be due to their obligatory investment in raising offspring, such as pregnancy and 
lactation (Trivers, 1972). Thus, women require more resources to ensure the survival of themselves and their offspring (Buss \& Schmitt, 1993; Kenrick \& Keefe, 1992).

The sex difference in mating strategy may also be the product of learning in modern societies. Physical attractiveness is widely thought to be socially desirable and thus both men and women seek physical attractiveness in mate choice. However, there is a large gap between men and women in the financial resources and social power they possess, despite the increasing gender equality across the globe. Many societies still have sex-differentiated stereotypes with men acting as resource providers and women acting as home-makers. The sex difference in mate preferences may well be shaped by both the actual gaps and the sex-stereotypical expectations (Eagly \& Wood, 1999). There is evidence suggesting that the sex differences of mate preferences tend to diminish in more balanced societies and environments (Finkel \& Eastwick, 2009; Walter et al., 2020).

Rational use of cognitive resources, such as the use of heuristic rules, also signals ecological rationality in mate choice. Our study considered the most widely discussed attributes in mate choice. However, mate choice in real life typically involves more complex information. In some cases, it might even be implausible to consider all relevant information. Individuals might adopt more cognitively efficient strategies, such as lexicographic rules, even if they may lead to intransitive mate preferences (Gigerenzer \& Goldstein, 1996; Mill \& Todd, 1998; Grammer et al., 2002). Rational decision making enables humans to adjust their mating strategies in response to different social environments and achieve good choices. The use of 
simple heuristics can be ecologically rational, even if they sometimes violate traditional standards of rationality such as transitivity (Todd et al., 2012; Lenton et al, 2013).

\section{Future work}

Our study has some limitations that could be addressed in future investigations. First, our construction of stimuli did not constitute a complete set of information involved in a typical mating decision. Although our study included the most pervasive and important attributes: physical attractiveness and financial resources, we did not include information such as age, occupation, and personality. Undoubtedly those factors shape mate preference, but what is probably more important is that the more complex information may alter participants' decision strategies (Bröder, 2000; Payne et al., 1988; Lenton et al, 2013). Lexicographic rules may be used more often in such more complex settings. On the other hand, the face images that conveyed physical attractiveness in the experiment may reveal other information such as perceived trustworthiness and health (Oosterhof \& Todorov, 2008; Wang et al., 2019; Little et al., 2011), although physical attractiveness is believed to be the principal information (Little et al., 2011). It would be interesting to decompose more relevant information from the face images and integrate them into the experimental test of transitivity. The transitivity of mate preference with more complex information remains an open question.

Second, we assumed that participants had monotonic preferences along each of the attributes. For example, we assumed that participants always preferred the more 
attractive candidate to the less attractive one, setting aside other attributes. Although this is a reasonable assumption, it has been argued that mate preferences sometimes may depend on the candidate's similarity to themselves (Buston \& Emlen, 2003).

Previous studies suggest that such a similarity-based rule is especially likely to appear when people verbally report their preferences, rather than in preferential choices (Todd et al., 2007). Our study uses preferential choice tasks and thus is less likely to induce such a similarity-based choice rule. Our monotonic assumption is also consistent with the weighted additive model that outperforms alternative models in Brandner et al. (2020). Yet, a closer examination of the monotonic assumption would be useful to further justify this assumption.

Third, our study was restricted to forced choice tasks. Another popular paradigm for experimental testing of transitivity is the binary choice with an indifference option (e.g., Hatz et al., 2020). This paradigm allows the usage of more general models of stochastic transitivity such as the Weak Order Mixture Model. The mixture model of transitive preference considered in our study is similar to the Weak Order Mixture Model, but it cannot be directly applied to binary choice with indifference option. We look forward to future investigations to test other transitive models with different experimental paradigms (e.g., Birnbaum, 2013).

Finally, future research can also extend this work to other principles of rational decision making. A good example is the independence of irrelevant alternatives (IIA) axiom, which suggests that the preference between two options should be independent of a third option (see e.g., Tversky \& Simonson, 1993). In the 
context of mate choice, there are likely more than two possible alternatives under consideration. Thus a test of independence (and its violations) would be valuable. Studies have found some evidence against IIA both in non-human animals (Lea \& Ryan, 2015) and humans (Sedikides et al., 1999), while few have formalized such a test . Further research can test the independence axiom in mate choice by using systematic evaluation and comparisons of the mathematical models. We hope that future research can extend the decision-theoretic approach to foster a better understanding of human mate preferences. 


\section{References}

Arbuthnott, D., Fedina, T. Y., Pletcher, S. D., \& Promislow, D. E. (2017). Mate choice in fruit flies is rational and adaptive. Nature Communications, 8(1), 1-9.

Bhatia, S., \& Loomes, G. (2017). Noisy preferences in risky choice: A cautionary note. Psychological Review, 124(5), 678-687.

Birnbaum, M. H. (2013). True-and-error models violate independence and yet they are testable. Judgment and Decision Making, 8(6), 717-737.

Birnbaum, M. H., \& Gutierrez, R. J. (2007). Testing for intransitivity of preferences predicted by a lexicographic semi-order. Organizational Behavior and Human Decision Processes, 104(1), 96-112.

Birnbaum, M. H., \& Schmidt, U. (2008). An experimental investigation of violations of transitivity in choice under uncertainty. Journal of Risk and Uncertainty, 37(1), 77-91.

Birnbaum, M. H., \& Schmidt, U. (2010). Testing transitivity in choice under risk. Theory and Decision, 69(4), 599-614.

Brandner, J. L., Brase, G. L., \& Huxman, S. A. (2020). "Weighting" to find the right person: compensatory trait integrating versus alternative models to assess mate value. Evolution and Human Behavior, 41(4), 284-292.

Broomell, S. B., Budescu, D. V. \& Por, H. (2011). Pair-wise comparisons of multiple models. Judgment \& Decision Making, 6(8), 821-831. 
Bröder, A. (2000). Assessing the empirical validity of the "Take-The-Best" heuristic as a model of human probabilistic inference. Journal of Experimental Psychology: Learning, Memory, and Cognition, 26, 1332-1346.

Buss, D. M. (1989). Sex differences in human mate preferences: Evolutionary hypotheses tested in 37 cultures. Behavioral and brain sciences, 12(1), 1-14.

Buss, D. M., \& Barnes, M. (1986). Preferences in human mate selection. Journal of personality and social psychology, 50(3), 559-570.

Buss, D. M., \& Schmitt, D. P. (1993). Sexual strategies theory: an evolutionary perspective on human mating. Psychological Review, 100(2), 204-232.

Buss, D. M., Shackelford, T. K., Kirkpatrick, L. A., \& Larsen, R. J. (2001). A half century of mate preferences: The cultural evolution of values. Journal of Marriage and Family, 63(2), 491-503.

Buston, P. M., \& Emlen, S. T. (2003). Cognitive processes underlying human mate choice: The relationship between self-perception and mate preference in Western society. Proceedings of the National Academy of Sciences, 100(15), 8805-8810.

Cavagnaro, D. R., \& Davis-Stober, C. P. (2014). Transitive in our preferences, but transitive in different ways: An analysis of choice variability. Decision, 1(2), 102-122.

Conroy-Beam, D. (2018). Euclidean mate value and power of choice on the mating market. Personality and Social Psychology Bulletin, 44(2), 252-264. 
Conroy-Beam, D., \& Buss, D. M. (2016). How are mate preferences linked with actual mate selection? Tests of mate preference integration algorithms using computer simulations and actual mating couples. PloS One, 11(6), e0156078.

Conroy-Beam, D., \& Buss, D. M. (2017). Euclidean distances discriminatively predict short-term and long-term attraction to potential mates. Evolution and Human Behavior, 38(4), 442-450.

Dai, J. (2017). Are intertemporal preferences transitive? A Bayesian analysis of repeated individual intertemporal choices. Decision, 4(1), 1-24.

Davis-Stober, C. P. (2012). A lexicographic semiorder polytope and probabilistic representations of choice. Journal of Mathematical Psychology, 56(2), 86-94.

Davis-Stober, C. P., Brown, N., \& Cavagnaro, D. R. (2015). Individual differences in the algebraic structure of preferences. Journal of Mathematical Psychology, 66, 70-82.

Davis-Stober, C. P., McCarthy, D. M., Cavagnaro, D. R., Price, M., Brown, N., \& Park, S. (2019). Is cognitive impairment related to violations of rationality? A laboratory alcohol intoxication study testing transitivity of preference. Decision, 6(2), 134-144.

Dechaume-Moncharmont, F. X., Freychet, M., Motreuil, S., \& Cézilly, F. (2013). Female mate choice in convict cichlids is transitive and consistent with a selfreferent directional preference. Frontiers in Zoology, 10(1), 69-78.

Diener, E., \& Seligman, M. E. (2002). Very happy people. Psychological Science, 13(1), 81-84. 
Falahatgar, M., Jain, A., Orlitsky, A., Pichapati, V., \& Ravindrakumar, V. (2018, July). The limits of maxing, ranking, and preference learning. In International Conference on Machine Learning (pp. 1427-1436). PMLR.

Eagly, A. H., \& Wood, W. (1999). The origins of sex differences in human behavior: Evolved dispositions versus social roles. American Psychologist, 54(6), 408-423.

Feingold, A. (1990). Gender differences in effects of physical attractiveness on romantic attraction: A comparison across five research paradigms. Journal of Personality and Social Psychology, 59(5), 981-993.

Feingold, A. (1992). Gender differences in mate selection preferences: A test of the parental investment model. Psychological Bulletin, 112(1), 125-139.

Finkel, E. J., \& Eastwick, P. W. (2009). Arbitrary social norms influence sex differences in romantic selectivity. Psychological Science, 20(10), 1290-1295.

Fishburn, P. C. (1973). Binary choice probabilities: on the varieties of stochastic transitivity. Journal of Mathematical Psychology, 10(4), 327-352.

Gigerenzer, G., \& Goldstein, D. G. (1996). Reasoning the fast and frugal way: Models of bounded rationality. Psychological Review, 103(4), 650-669.

Gangestad, S. W., \& Simpson, J. A. (2000). The evolution of human mating: Tradeoffs and strategic pluralism. Behavioral and Brain Sciences, 23, 573-587.

Grammer, K., Fink, B., Juette, A., Ronzal, G., \& Thornhill, R. (2002). Female faces and bodies: N-dimensional feature space and attractiveness. In G. Rhodes \& L. A. Zebrowitz (Eds.), Advances in visual cognition, Vol. 1. Facial attractiveness: Evolutionary, cognitive, and social perspectives (p. 91-125). Ablex Publishing. 
Guo, Y. (2018). Rationality or irrationality of preferences? Quantitative tests of decision theories (Doctoral dissertation, University of Illinois at UrbanaChampaign).

Hatz, L. E., Park, S., McCarty, K. N., McCarthy, D. M., \& Davis-Stober, C. P. (2020). Young adults make rational sexual decisions. Psychological Science, 31(8), 944956.

He, L., Golman, R., \& Bhatia, S. (2019). Variable time preference. Cognitive Psychology, 111, 53-79.

He, L., Zhao, W. J., \& Bhatia, S. (2020). An ontology of decision models. Psychological Review, 10.1037/rev0000231. Advance online publication.

Hehman, E., Sutherland, C. A., Flake, J. K., \& Slepian, M. L. (2017). The unique contributions of perceiver and target characteristics in person perception. Journal of Personality and Social Psychology, 113(4), 513-529.

Hill, R. (1945). Campus values in mate-selection. Journal of Home Economics, 37, $554-558$.

Howard, J. A., Blumstein, P., \& Schwartz, P. (1987). Social or evolutionary theories? Some observations on preferences in human mate selection. Journal of Personality and Social Psychology, 53(1), 194-200.

Huber, J., Payne, J. W., \& Puto, C. (1982). Adding asymmetrically dominated alternatives: Violations of regularity and the similarity hypothesis. Journal of Consumer Research, 9(1), 90-98. 
Kahneman, D., \& Tversky, A. (1979). Prospect Theory: An Analysis of Decision under Risk. Econometrica, 47(2), 263-292.

Kass, R. E., \& Raftery, A. E. (1995). Bayes factors. Journal of the American Statistical Association, 90(430), 773-795.

Kenrick, D. T., Groth, G. E., Trost, M. R., \& Sadalla, E. K. (1993). Integrating evolutionary and social exchange perspectives on relationships: Effects of gender, self-appraisal, and involvement level on mate selection criteria. Journal of Personality and Social Psychology, 64, 951-969.

Kenrick, D. T., \& Keefe, R. C. (1992). Age preferences in mates reflect sex differences in human reproductive strategies. Behavioral and Brain Sciences, 15(1), 75-91.

Klugkist, I., \& Hoijtink, H. (2007). The Bayes factor for inequality and about equality constrained models. Computational Statistics \& Data Analysis, 51(12), 63676379.

Lea, A. M., \& Ryan, M. J. (2015). Irrationality in mate choice revealed by túngara frogs. Science, 349(6251), 964-966.

Lenton, A.P., Penke, L., Todd, P.M., and Fasolo, B. (2013). The heart has its reasons: Social rationality in mate choice. In R. Hertwig, U. Hoffrage, and the ABC Research Group, Simple heuristics in a social world (pp. 433-457). Oxford University Press. 
Li, N. P., Bailey, J. M., Kenrick, D. T., \& Linsenmeier, J. A. (2002). The necessities and luxuries of mate preferences: testing the tradeoffs. Journal of personality and social psychology, 82(6), 947-955.

Li, N. P., \& Kenrick, D. T. (2006). Sex similarities and differences in preferences for short-term mates: What, whether, and why. Journal of Personality and Social Psychology, 90, 468-489.

Li, N. P., Valentine, K. A., \& Patel, L. (2011). Mate preferences in the US and Singapore: A cross-cultural test of the mate preference priority model. Personality and Individual Differences, 50(2), 291-294.

Lieder, F., \& Griffiths, T. L. (2017). Strategy selection as rational metareasoning. Psychological Review, 124(6), 762-794.

Little, A. C., Jones, B. C., \& DeBruine, L. M. (2011). Facial attractiveness: evolutionary based research. Philosophical Transactions of the Royal Society B: Biological Sciences, 366(1571), 1638-1659.

Loomes, G., \& Sugden, R. (1995). Incorporating a stochastic element into decision theories. European Economic Review, 39, 641-648.

Luce, R. D. (1959). Individual choice behavior: A theoretical analysis. New York: Wiley.

Maestripieri, D., Henry, A., \& Nickels, N. (2017). Explaining financial and prosocial biases in favor of attractive people: Interdisciplinary perspectives from economics, social psychology, and evolutionary psychology. Behavioral and Brain Sciences, 40, E19. 
Miller, G.F., \& Todd, P.M. (1998). Mate choice turns cognitive. Trends in Cognitive Sciences, 2, 190-198.

Oosterhof, N. N., \& Todorov, A. (2008). The functional basis of face evaluation. Proceedings of the National Academy of Sciences, 105(32), 1108711092.

Payne, J. W., Bettman, J. R., \& Johnson, E. J. (1988). Adaptive strategy selection in decision making. Journal of experimental psychology: Learning, Memory, and Cognition, 14(3), 534-552.

Payne, J. W., Bettman, J. R., \& Johnson, E. J. (1993). The Adaptive Decision Maker. Cambridge, England: Cambridge University Press.

Raftery, A. E. (1996). Approximate Bayes factors and accounting for model uncertainty in generalised linear models. Biometrika, 83(2), 251-266.

Regenwetter, M., Dana, J., \& Davis-Stober, C. P. (2010). Testing transitivity of preferences on two-alternative forced choice data. Frontiers in psychology, 1 , 148.

Regenwetter, M., Dana, J., \& Davis-Stober, C. P. (2011). Transitivity of preferences. Psychological Review, 118(1), 42-56.

Roelofsma, P. H., \& Read, D. (2000). Intransitive intertemporal choice. Journal of Behavioral Decision Making, 13(2), 161-177.

Sedikides, C., Ariely, D., \& Olsen, N. (1999). Contextual and procedural determinants of partner selection: Of asymmetric dominance and prominence. Social Cognition, 17(2), 118-139. 
Shafir, S. (1994). Intransitivity of preferences in honey bees: support for 'comparative' evaluation of foraging options. Animal Behaviour, 48(1), 55-67.

Simão, J., \& Todd, P. M. (2002). Modeling mate choice in monogamous mating systems with courtship. Adaptive Behavior, 10(2), 113-136

Simonson, I. (1989). Choice based on reasons: The case of attraction and compromise effects. Journal of Consumer Research, 16(2), 158-174.

Sopher, B., \& Gigliotti, G. (1993). Intransitive cycles: Rational Choice or random error? An answer based on estimation of error rates with experimental data. Theory and decision, 35(3), 311-336.

Symons, D. (1979). The evolution of human sexuality. New York: Oxford University Press.

Stan Development Team (2020). RStan: The R interface to Stan. R package version 2.18.2. http://mc-stan.org/.

Todd, P.M., Gigerenzer, G., and the ABC Research Group. (2012). Ecological rationality: Intelligence in the world. Oxford University Press.

Todd, P. M., \& Miller, G. F. (1999). From pride and prejudice to persuasion: Satisficing in mate search. In G. Gigerenzer, P. M. Todd \& the ABC Research Group (Eds.), Simple heuristics that make us smart (pp. 287-308). New York: Oxford University Press.

Todd, P. M., Penke, L., Fasolo, B., \& Lenton, A. P. (2007). Different cognitive processes underlie human mate choices and mate preferences. Proceedings of the National Academy of Sciences, 104(38), 15011-15016. 
Tversky, A. (1969). Intransitivity of preferences. Psychological Review, 76(1), 31-48.

Tversky, A., \& Simonson, I. (1993). Context-dependent preferences. Management Science, 39(10), 1179-1189.

Trivers, R. L. (1972). Parental investment and sexual selection. In B. Campbell (Ed.), Sexual Selection and the Descent of Man (pp. 136-179). Aldinc: Chicago.

Walter, K. V., Conroy-Beam, D., Buss, D. M., Asao, K., Sorokowska, A., Sorokowski, P., ... \& Amjad, N. (2020). Sex differences in mate preferences across 45 countries: A large-scale replication. Psychological Science, 31(4), 408423.

Zhang, L., Wang, H., Lee, A. J., DeBruine, L. M., \& Jones, B. C. (2019). Chinese and UK participants' preferences for physical attractiveness and social status in potential mates. Royal Society open science, 6(11), 181243. 